\title{
MOMENT MAPS AND RIEMANNIAN SYMMETRIC PAIRS
}

\author{
LUIS O'SHEA AND REYER SJAMAAR*
}

\begin{abstract}
We study Hamiltonian actions of a compact Lie group on a symplectic manifold in the presence of an involution on the group and an antisymplectic involution on the manifold. The fixed-point set of the involution on the manifold is a Lagrangian submanifold. We investigate its image under the moment map and conclude that the intersection with the Weyl chamber is an easily described subpolytope of the Kirwan polytope. Of special interest is the integral Kähler case, where much stronger results hold. In particular, we obtain convexity theorems for closures of orbits of the noncompact dual group (in the sense of the theory of symmetric pairs). In the abelian case these results were obtained earlier by Duistermaat. We derive explicit inequalities for polytopes associated with real flag varieties.
\end{abstract}

\section{Contents}

\begin{tabular}{|lr|}
\hline 1. & Introduction \\
\hline $2 . \quad$ Elementary properties and examples & 1 \\
\hline 3. The main result & 2 \\
\hline 4. Geometric quantization & 7 \\
\hline 5. The projective case & 9 \\
\hline $6 . \quad$ The affine cas & 15 \\
\hline $7 . \quad$ Normal forms & 20 \\
\hline 8. Proof of the main theorem & 22 \\
\hline $9 . \quad$ Applications & 24 \\
\hline Appendix A. Anti-symplectic maps & 25 \\
\hline Appendix B. Symmetric pairs & 31 \\
\hline Appendix C. Notation & 32 \\
\hline References & 34 \\
\hline
\end{tabular}

\section{INTRODUCTION}

The main result of this paper is a "real form" of Kirwan's convexity theorem. We apply our result to flag varieties of real semisimple groups and thus obtain eigenvalue inequalities, which generalize inequalities found by Weyl, Ky Fan, Kostant, Klyachko, and many others. For instance, we find an answer to the following question: given the singular values of two rectangular matrices (of the same size), what are the possible singular values of their sum?

Date: 8 February 1999. Revised 25 October 1999.

*Partially supported by an Alfred P. Sloan Research Fellowship and by NSF Grant DMS9703947. 
We obtain these results by studying symplectic manifolds $M$ equipped with an antisymplectic involution $\tau$ and a Hamiltonian action of a compact Lie group $U$. We assume the fixed-point set of the involution to be nonempty, in which case it is a Lagrangian submanifold. We denote the moment map for the $U$-action on $M$ by $\Phi$. Our goal is to describe the image of the Lagrangian under $\Phi$. Good results can of course only be expected if the involution and the action are related in a reasonable way. The right condition to impose is for $\Phi$ to be "anti-equivariant" relative to the involution $\tau$ and an involutive automorphism $\sigma$ on the group $U$. The Lagrangian is then not stable under the action of $U$, but only under the fixed-point group $U^{\sigma}$. Thus the theory of Riemannian symmetric pairs comes into play.

If $U$ is a torus and $\sigma$ is inversion, anti-equivariance amounts to invariance of $\Phi$ with respect to $\tau$. This special case was studied by Duistermaat [6], who proved that the image of $M^{\tau}$ under $\Phi$ is equal to the full image $\Phi(M)$.

This turns out to be false in general. Our main result is the following description of $\Phi\left(M^{\tau}\right)$ as a subset of $\Phi(M)$. Instead of the image $\Phi(M)$, it is easier to consider the intersection $\Delta(M)=\Phi(M) \cap \mathfrak{t}_{+}^{*}$, where $\mathfrak{t}_{+}^{*}$ is a Weyl chamber in $\mathfrak{u}^{*}$. According to Kirwan, $\Delta(M)$ is a convex polytope, called the moment polytope of $M$. Our result is that the set $\Delta\left(M^{\tau}\right)=\Phi\left(M^{\tau}\right) \cap \mathfrak{t}_{+}^{*}$ is equal to the subpolytope of $\Delta(M)$ consisting of symmetric elements. In other words, $\Delta\left(M^{\tau}\right)$ is equal to the intersection of $\Delta(M)$ with the Weyl chamber of the restricted root system.

The proof is an adaptation of the proof of Kirwan's convexity theorem given in 28. (We also attempted to adapt the perhaps simpler approach followed by Lerman et al. [21], but were unable to do so.) Briefly, the method is to establish the result first in the Kähler case (more precisely, for projective and affine varieties), and then handle the general case by dint of a local normal form theorem, which asserts that near the orbits that matter every Hamiltonian $U$-manifold is isomorphic to an affine variety in a suitable sense. An advantage of this approach is that it yields a characterization of the vertices and an attractive, if unwieldy, invariant-theoretic local description of the polytope near any point. More importantly, the Kähler case has the noteworthy feature that $G$, the noncompact real reductive group dual to $U$, acts holomorphically and commutes with the antiholomorphic involution $\tau$. We are thus led to a convexity theorem for closures of $G$-orbits, which is a nonabelian version of a result of Atiyah's.

In Section 2 we introduce the category of Hamiltonian $(U, \sigma)$-manifolds and give a number of examples. Our main result is stated in Section 3. Section 4 is an exposé of Kähler quantization for Hamiltonian $(U, \sigma)$-manifolds, where we develop the techniques needed for establishing the convexity theorem in the Kähler case, which we carry out in Sections 5 and 6. We then state the normal form theorems in Section 7 and finish the proof in Section 8 . Applications inspired by mechanics (cotangent bundles) and representation theory (coadjoint orbits) are discussed in

Section 9. A few elementary results on antisymplectic maps and symmetric pairs are covered in Appendices $\mathrm{A}$ and B. Appendix $\mathrm{C}$ is a list of notational conventions.

\section{Elementary properties AND EXAmples}

Let $U$ be a compact Lie group. Let $\sigma$ be an involution of $U$, i.e. an automorphism which satisfies $\sigma^{2}=\mathrm{id}_{U}$. (We are mostly interested in the case where $\sigma$ is not the identity.) The derivative of $\sigma$ induces involutions on the Lie algebra $\mathfrak{u}$ and on the dual space $\mathfrak{u}^{*}$, both of which we denote for simplicity also by $\sigma$. 
2.1. Definition. A Hamiltonian $(U, \sigma)$-manifold is a quadruple $(M, \omega, \tau, \Phi)$ consisting of a smooth manifold $M$ equipped with an action of the group $U$, a symplectic form $\omega$, an antisymplectic involution $\tau$ (i.e. a diffeomorphism $\tau$ satisfying $\tau^{2}=\operatorname{id}_{M}$ and $\left.\tau^{*} \omega=-\omega\right)$, and a $U$-equivariant map $\Phi: M \rightarrow \mathfrak{u}^{*}$ which is a moment map for the action of $U$. In addition we impose the following two conditions:

$$
\begin{gathered}
\Phi(\tau(m))=-\sigma(\Phi(m)), \\
\tau(u m)=\sigma(u) \tau(m)
\end{gathered}
$$

for all $m \in M$ and $u \in U$.

Consider the semidirect product $U \rtimes\{ \pm 1\}$, where the action of $\{ \pm 1\}$ on $U$ is given by the involution $\sigma$. (By $\{ \pm 1\}$ we mean a copy of $\mathbb{Z} / 2$ written multiplicatively.) Then (2.2) says that $U \rtimes\{ \pm 1\}$ acts on $M$ and that the action is sesquisymplectic in the sense that $u^{*} \omega=\varepsilon(u) \omega$, where $\varepsilon: U \rtimes\{ \pm 1\} \rightarrow\{ \pm 1\}$ denotes the canonical homomorphism.

Properties (2.1) and (2.2) are not independent. For $\xi \in \mathfrak{u}$ we denote by $\xi_{M}$ the induced vector field on $M$ and by $\Phi^{\xi}$ the function on $M$ defined by $\Phi^{\xi}(m)=$ $\langle\Phi(m), \xi\rangle$.

2.2. Lemma. If $U$ is connected, then (2.1) implies (2.2). Conversely, if (2.2) is satisfied, we can shift $\Phi$ by a suitable constant to ensure that (2.1) holds.

Proof. Using the moment map condition $d \Phi^{\xi}=\iota\left(\xi_{M}\right) \omega$ we obtain from (2.1) that

$$
\tau_{*}\left(\xi_{M}\right)=(\sigma(\xi))_{M}
$$

for all $\xi \in \mathfrak{u}$. This implies (2.2) if $U$ is connected. Conversely, if (2.2) holds then (2.3) holds, whether or not $U$ is connected. Therefore

$$
\begin{array}{r}
\langle d \Phi, \xi\rangle=\iota\left(\xi_{M}\right) \omega=\iota\left(\tau_{*} \sigma(\xi)_{M}\right) \omega=-\iota\left(\tau_{*} \sigma(\xi)_{M}\right) \tau^{*} \omega=-\tau^{*}\left(\iota\left(\sigma(\xi)_{M}\right) \omega\right) \\
=-\tau^{*}\langle d \Phi, \sigma(\xi)\rangle=-\langle d(\Phi \circ \tau), \sigma(\xi)\rangle=-\langle d(\sigma \circ \Phi \circ \tau), \xi\rangle
\end{array}
$$

for all $\xi$ and so $d(\sigma \circ \Phi \circ \tau)=-d \Phi$. Furthermore, it is easy to check that $\sigma \circ \Phi \circ \tau$ is $U$-equivariant. It follows that

$$
\sigma \circ \Phi \circ \tau=-\Phi+c
$$

for some $U$-invariant $c \in \mathfrak{u}^{*}$. Multiplying by $\sigma$ on the left and by $\tau$ on the right we get $\Phi=-\sigma \circ \Phi \circ \tau+\sigma(c)$, and therefore $\sigma(c)=c$. Putting $\Phi^{\prime}=\Phi+c^{\prime}$, with $c^{\prime}=-c / 2$, we see that $\Phi^{\prime}$ is a $U$-equivariant moment map satisfying

$$
\sigma \circ \Phi^{\prime} \circ \tau=\sigma \circ\left(\Phi+c^{\prime}\right) \circ \tau=\sigma \circ \Phi \circ \tau+c^{\prime}=-\Phi+c+c^{\prime}=-\Phi^{\prime},
$$

i.e. $\Phi^{\prime} \circ \tau=-\sigma \circ \Phi^{\prime}$.

Henceforth we assume $U$ to be connected, apart from a few exceptional situations where for technical reasons we need to allow disconnected groups. These exceptions will be pointed out explicitly.

The focus of our attention is the fixed-point manifold $M^{\tau}$. Let $K$ be the identity component of the fixed-point group $U^{\sigma}$ and let $\mathfrak{q} \subseteq \mathfrak{u}$ be the -1-eigenspace of $\sigma$. Then $\mathfrak{u}=\mathfrak{k} \oplus \mathfrak{q}$, where $\mathfrak{k}=\operatorname{Lie}(K)$. Identifying $\mathfrak{k}^{*}$ with the annihilator of $\mathfrak{q}$ and $\mathfrak{q}^{*}$ with the annihilator of $\mathfrak{k}$, we have $\mathfrak{u}^{*}=\mathfrak{k}^{*} \oplus \mathfrak{q}^{*}$. Choose an almost complex structure $J$ on $M$ which is $U$-invariant and $\tau$-anti-invariant. The existence of such a $J$ is guaranteed by Lemma A.3(i) in Appendix A. 
2.3. Proposition. (i) If $M^{\tau}$ is nonempty, it is a $U^{\sigma}$-stable totally real Lagrangian submanifold of $M$, and $\Phi\left(M^{\tau}\right)$ is contained in $\mathfrak{q}^{*}$.

(ii) Every connected component of $M^{\tau}$ is $K$-stable.

(iii) The $K$-orbit through every $m$ in $M^{\tau}$ is a connected component of $U m \cap M^{\tau}$.

Proof. The inclusion $\Phi\left(M^{\tau}\right) \subseteq \mathfrak{q}^{*}$ is obvious from (2.1) and the $U^{\sigma}$-stability of $M^{\tau}$ is obvious from (2.2). The fact that $M^{\tau}$ is Lagrangian and totally real follows from Lemma A.3 (iii) (applied to the group $\mathcal{G}=U \rtimes\{ \pm 1\}$ ). This proves (ii).

(iii) follows immediately from (ii) and the fact that $K$ is connected.

Let $m \in M^{\tau}$. It follows from (iii) that $K m \subseteq U m \cap M^{\tau}$. To prove (iii) it suffices to show that $K m$ is open in $U m \cap M^{\tau}$, or equivalently that the tangent space to $K m$ at $m$ is equal to $T_{m}(U m) \cap T_{m} M^{\tau}$. A general tangent vector to $U m$ at $m$ is of the form $\xi_{M}=\xi_{M}^{+}+\xi_{M}^{-}$with $\xi^{+} \in \mathfrak{k}$ and $\xi^{-} \in \mathfrak{q}$. Then $\tau_{*}\left(\xi_{M}\right)=\sigma(\xi)_{M}=\xi_{M}^{+}-\xi_{M}^{-}$, so $\xi_{M}$ is tangent to $M^{\tau}$ if and only if $\tau_{*}\left(\xi_{M}\right)=\xi_{M}$ if and only if $\xi_{M}^{-}=0$, i.e. $\xi_{M}=\xi_{M}^{+} \in T_{m}(K m)$.

2.4. Example (tori). Let $U$ be a torus and $\sigma$ the involution which sends $u$ to $u^{-1}$. Then $U^{\sigma}$ is the subgroup of 2-torsion elements of $U$ and condition (2.1) boils down to $\Phi$ being invariant under $\tau$. This is the setting of Duistermaat's paper [6].

2.5. Example (connections). This infinite-dimensional example was suggested to us by C. Woodward. Consider an oriented Riemann surface $\Sigma$ and an orientationreversing involution $f: \Sigma \rightarrow \Sigma$ without fixed points. Let $P$ be a trivial principal $U$-bundle over $\Sigma$ and $\mathfrak{A}$ the space of flat connections on $P$. According to Atiyah and Bott $\mathfrak{A}$ is a Hamiltonian $\mathfrak{U}$-manifold, where $\mathfrak{U}=C^{\infty}(\Sigma, U)$ is the group of gauge transformations. Involutions on $\mathfrak{A}$ and $\mathfrak{U}$ are given by pullback: $\tau(A)=f^{*} A$ and $\sigma(u)=f^{*} u$. Then $\tau$ is antisymplectic because $f$ reverses the orientation and $\sigma$ is a group homomorphism. One checks easily that $\mathfrak{A}$ is a Hamiltonian $(\mathfrak{U}, \sigma)$ manifold. Moreover, the Lagrangian $\mathfrak{A}^{\tau}$ is isomorphic to the space of connections on the quotient bundle $P / f$ over the non-orientable quotient surface $\Sigma / f$, and $\mathfrak{U}^{\sigma}$ is the gauge group $C^{\infty}(\Sigma / f, U)$.

2.6. Example (cotangent bundles). Let $S$ be any manifold with an involution $\tau$ and let $M=T^{*} S$ be the cotangent bundle of $S$, equipped with its standard symplectic form. The cotangent lift of $\tau$ is a symplectic involution of $M$. We turn it into an antisymplectic involution, also called $\tau$, by "reversing momenta", i.e. by multiplying by -1 in the cotangent directions. The fixed-point set $M^{\tau}$ is equal to the conormal bundle of $S^{\tau}$. Suppose that $U$ acts on $S$ in such a way that $\tau(u s)=\sigma(u) \tau(s)$ for $s \in S$, and lift the action to $M$ in the canonical way. It is then a straightforward exercise to check that $M$ is a Hamiltonian $(U, \sigma)$-manifold. The moment map is given by $\Phi^{\xi}(s, p)=p\left(\xi_{S, s}\right)$ for $\xi \in \mathfrak{u}, s \in S$ and $p \in T_{s}^{*} S$. For instance, we can take $S$ to be the group $U$ with involution $\sigma$. If we identify $M=T^{*} U$ with $U \times \mathfrak{u}^{*}$ by means of left translations, then $\tau$ is given by $\tau(u, \xi)=(\sigma(u),-\sigma(\xi))$, and $M^{\tau}=U^{\sigma} \times \mathfrak{q}^{*}$. On $M$ there are three different Hamiltonian $U$-actions, namely the actions induced by left multiplication, right multiplication, and conjugation. Thus $M$ is a Hamiltonian $(U, \sigma)$-manifold in three different ways.

2.7. Example (vector spaces). In this example $U$ need not be connected. Let $(V, \omega)$ be a finite-dimensional symplectic vector space and let $\tau \in \mathbf{G} \mathbf{L}(V)$ be an 
antisymplectic involution. Assume that $U$ acts linearly and symplectically on $V$ and that (2.2) holds for all $u \in U$ and $m \in V$. Let $\Phi_{V}$ be the standard quadratic moment map for the $U$-action given by

$$
\Phi_{V}^{\xi}(v)=\frac{1}{2} \omega(\xi v, v) .
$$

Then the quadruple $\left(V, \omega, \tau, \Phi_{V}\right)$ is a Hamiltonian $(U, \sigma)$-manifold, i.e. (2.1) holds automatically. The fixed-point set $V^{\tau}$ is clearly nonempty and is in fact a $U^{\sigma_{-}}$ stable Lagrangian subspace. Furthermore, by Lemma A.1 there exists a $U$-invariant and $\tau$-anti-invariant compatible complex structure $J$ on $V$. Then we can define a real inner product $\beta$ on $V$ by $\beta(v, w)=\omega(v, J w)$ and a Hermitian inner product $h$ by $h=\beta+i \omega$. Clearly $h$ is $U$-invariant and $h(\tau(v), \tau(w))=h(w, v)$. As a unitary $U^{\sigma}$-module (but not as a $U$-module) $V$ is canonically isomorphic to the complexification of $V^{\tau}$, and $\tau$ is complex conjugation relative to the real form $V^{\tau}$. Let $\mathcal{A}: U \rightarrow \mathbf{U}(V)$ be the homomorphism defining the action of $U$ on $V$. We call the data $(V, \omega, \tau, J, \mathcal{A})$ (often abbreviated to $V)$ a unitary $(U, \sigma)$-module. See Section 1 for a geometric construction and a classification of such modules.

2.8. Remark. Let $V$ be a unitary $(U, \sigma)$-module. Consider the unitary group $\mathbf{U}(V)$ and the standard involution on $\mathbf{U}(V)$ defined by $\sigma_{V}(x)=\left(x^{-1}\right)^{t}$, where " $t$ " denotes transpose with respect to the Hermitian form $h$. It follows from (2.2) that the representation $\mathcal{A}$ intertwines the involutions on $U$ and $\mathbf{U}(V): \mathcal{A} \sigma=\sigma_{V} \mathcal{A}$. In fact, this property can be taken as an alternative definition of a unitary $(U, \sigma)$ module. Here is yet another, purely complex algebraic characterization. Define

$$
b(v, w)=h(v, \tau(w)) .
$$

Then $b$ is a nondegenerate symmetric complex-bilinear form and is "twisted" invariant under $U$ in the sense that $b(u v, \sigma(u) w)=b(v, w)$ for all $u \in U$ and $v$, $w \in V$. Conversely, a $U$-module furnished with such a form can be endowed with the structure of a unitary $(U, \sigma)$-module such that (2.5) holds.

2.9. Example (coadjoint orbits). Let $\lambda \in \mathfrak{u}^{*}$ and let $M=U \lambda$ be the coadjoint orbit through $\lambda$, equipped with its standard symplectic form. Assume that $-\sigma(\lambda) \in$ $M$, so that $-\sigma(M)=M$. Then we can define an involution $\tau$ on $M$ by putting $\tau(x)=-\sigma(x)$; this is antisymplectic because $\sigma$ is a Lie algebra homomorphism. The moment map is simply the inclusion of $M$ into $\mathfrak{u}^{*}$, and therefore (2.1) holds by construction. Note that the fixed-point set $M^{\tau}$ is equal to $M \cap \mathfrak{q}^{*}$, so if it is nonempty we may assume that $\lambda$ is in $\mathfrak{q}^{*}$. We call the coadjoint orbit $M$ symmetric if it is of the form $U \lambda$ with $\lambda \in \mathfrak{q}^{*}$. We assert that if $\lambda \in \mathfrak{q}^{*}$ then $M^{\tau}$ is a single $K$-orbit,

$$
M^{\tau}=U^{\sigma} \lambda=K \lambda .
$$

To prove this we invoke Proposition 2.3, according to which it is enough to show that $M^{\tau}$ is connected. Observe that the involution on $\mathfrak{u}$ maps $[\mathfrak{u}, \mathfrak{u}]$ into itself. Let $\bar{U}$ be any connected Lie group with Lie algebra $[\mathfrak{u}, \mathfrak{u}]$ such that the involution on $\mathfrak{u}$ descends to an involution $\bar{\sigma}$ on $\bar{U}$. Then $\bar{U}$ acts on $\mathfrak{u}$ and $\mathfrak{u}^{*}$, and the orbits of $\bar{U}$ are the same as those of $U$. It follows from the proof of Proposition 8.8(ii) in Helgason [12, Ch. VII] that $M^{\tau}=\bar{U}^{\bar{\sigma}} \lambda$ for any such $\bar{U}$. We can take $\bar{U}$ to be the universal cover of $[U, U]$, in which case the group $\bar{U}^{\bar{\sigma}}$ is connected by Theorem 8.2 of [12, Ch. VII]. The upshot is that $M^{\tau}=\bar{U}^{\bar{\sigma}} \lambda$ is connected, which concludes the proof of (2.6). This example is continued in 3.4, 4.8, 5.6, 5.14, and in Section 9 . 
2.10. Example (symplectic quotients). Let $M$ be a Hamiltonian $(U, \sigma)$-manifold and let $H$ be a Lie group which acts on $M$ in a Hamiltonian fashion with momentum map $\Psi: M \rightarrow \mathfrak{h}^{*}$. Assume that the action of $H$ commutes with that of $U$. Assume also that $\tau$ maps $\Psi^{-1}(0)$ into itself and maps $H$-orbits to $H$-orbits, i.e. for all $h \in H$ and $m \in M$ there exists $h^{\prime} \in H$ such that $\tau(h m)=h^{\prime} \tau(m)$. (A sufficient condition for this to hold is that $H$ should be connected and that for every $\eta \in \mathfrak{h}$ there should exist $\eta^{\prime}$ such that $\tau^{*}\left(\Psi^{\eta}\right)=\Psi^{\eta^{\prime}}$.) Assume furthermore that $H$ acts properly and freely on the fibre $\Psi^{-1}(0)$, so that the quotient $M / / H=\Psi^{-1}(0) / H$ is a well-defined symplectic manifold. The action of $U$ and the map $\tau$ descend to $M / / H$ and it is easy to see that $M / / H$ is a Hamiltonian $(U, \sigma)$-manifold.

2.11. Example (projective varieties). Let $V$ be a unitary $(U, \sigma)$-module as in Example 2.7. Taking $H=S^{1}$ in Example 2.10 and letting $H$ act by complex scalar multiplication on $V$, we conclude that the projective space $\mathbb{P} V=V / / S^{1}$ is a Hamiltonian $(U, \sigma)$-manifold in a natural way. Likewise, every nonsingular complex subvariety of $\mathbb{P} V$ which is stable under $U$ and $\tau$ inherits the structure of a Hamiltonian $(U, \sigma)$-manifold.

2.12. Example (toric varieties). Let $(U, \sigma)$ be as in Example 2.4, let $\Delta$ be a simple lattice polytope in $\mathfrak{u}^{*}$, and let $M$ be the symplectic toric variety ("Delzant space") associated with $\Delta$. As explained e.g. in [25], this space can be obtained from $T^{*} U$ as a symplectic quotient with respect to an appropriate subtorus of $U$ and therefore, by Examples 2.6 and 2.10, has the structure of a Hamiltonian $(U, \sigma)$-manifold. In fact, $M$ is a complex projective variety in a natural way and as such is defined over $\mathbb{Z}$ and hence over $\mathbb{R}$. It is not hard to see that the antisymplectic involution $\tau$ on $M$ is the same as complex conjugation. The real part $M^{\tau}$ of $M$ is nonempty and connected. See e.g. 15, where the cohomology of $M^{\tau}$ with $\mathbb{Z} / 2$-coefficients is computed.

2.13. Example. The product of a Hamiltonian $\left(U_{1}, \sigma_{1}\right)$-manifold and a Hamiltonian $\left(U_{2}, \sigma_{2}\right)$-manifold is a Hamiltonian $\left(U_{1} \times U_{2}, \sigma_{1} \times \sigma_{2}\right)$-manifold. Let $H$ be a $\sigma$-stable closed subgroup of $U$. For simplicity we denote the restriction of $\sigma$ to $H$ also by $\sigma$. Then every Hamiltonian $(U, \sigma)$-manifold inherits the structure of an $(H, \sigma)$-manifold. In particular, the product of two Hamiltonian $(U, \sigma)$-manifolds is a Hamiltonian $(U, \sigma)$-manifold with respect to the diagonal action. Observe that these facts are also true if the groups in question are not connected.

2.14. Example (duplication). Let $(M, \omega, \Phi)$ be any Hamiltonian $U$-manifold. Let $M^{-}$be the same manifold as $M$, but furnished with the opposite symplectic form $-\omega$. Define $M^{\text {dup }}=M \sqcup M^{-}$, the disjoint union of $M$ and $M^{-}$, and define $\tau$ to be the antisymplectic involution which interchanges the two components. It has obviously no fixed points. Extend the $U$-action and the moment map from $M$ to

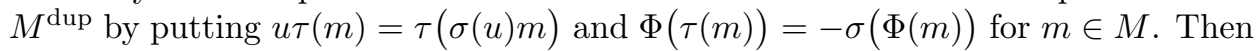
$M^{\text {dup }}$ is a Hamiltonian $(U, \sigma)$-manifold. For instance, if $M=U \lambda$ is a coadjoint orbit of $U$, then $M^{\text {dup }} \cong U \lambda \sqcup-\sigma(U \lambda)$.

2.15. Example. The process of duplication has linear analogue, which runs as follows. Let $V$ be any unitary $U$-module with Hermitian metric $h=\beta+i \omega$. Let $V^{-}$ be the vector space conjugate to $V$ equipped with the Hermitian form $\bar{h}=\beta-i \omega$. 
Put $V^{\text {dup }}=V \oplus V^{-}$and let $\tau$ be the map which interchanges the two components. It is obvious that $\tau$ is conjugate linear and antisymplectic. Turn $V^{-}$into a $U$ module by putting $u \tau(v)=\tau(\sigma(u) v)$ for $v \in V$; then $\left(V^{\text {dup }}, \tau\right)$ becomes a unitary $(U, \sigma)$-module as in Example 2.7. The fixed-point set of $\tau$ is the diagonal.

Here is a general criterion for the fixed-point set $M^{\tau}$ in a Hamiltonian $(U, \sigma)$ manifold $(M, \omega, \tau, \Phi)$ to be nonempty. The integrality condition is satisfied for instance if $M$ is prequantizable in the sense of Definition 4.1 .

2.16. Proposition. Suppose that $M$ is a compact connected $(U, \sigma)$-manifold of dimension $4 n$ and that there exists a class $c \in H^{2}(M, \mathbb{Z})$ such that $\tau^{*} c=-c$ and the image of $c$ in $H^{2}(M, \mathbb{R})$ is equal to the cohomology class of $\omega$. Then $M^{\tau}$ is nonempty and $\left.b^{n}\right|_{M^{\tau}}$ is nonzero, where $b \in H^{2}(M, \mathbb{Z} / 2)$ denotes the reduction modulo 2 of $c$.

Proof. Observe that $\tau^{*} b^{n}=b^{n}$ and that $b^{2 n}$ is the mod 2 fundamental class of $M$. The result now follows from Theorem 7.4 of Bredon [4, Ch. VII].

We do not know of a good criterion for the fixed-point set to be connected. In Examples 2.7, 2.9 and 2.12 the total space $M$ is simply connected and the Lagrangian $M^{\tau}$ is connected. The same is true for $M=T^{*} U$ (as in Example 2.6), provided that $U$ is simply connected. But here is an example where $M$ is simply connected and $M^{\tau}$ is disconnected. Let $S$ be the two-sphere with the involution $\tau$ given by rotation through $\pi$ about the vertical axis, and let $U=\mathbf{S O}(3)$ act on $S$ in the usual way. Then $\tau(u s)=\sigma(u) \tau(s)$, where $\sigma$ is the inner involution of $U$ defined by the matrix

$$
\left(\begin{array}{ccc}
-1 & 0 & 0 \\
0 & -1 & 0 \\
0 & 0 & 1
\end{array}\right) .
$$

Let $M=T^{*} S$ as in Example 2.6. Then $M^{\tau}$ is the union of two Lagrangian planes. However, this example is noncompact. This leads to the following question.

2.17. Problem. Is $M^{\tau}$ connected whenever $M$ is compact and simply connected?

\section{The MAIN RESUlT}

Let $(M, \omega, \tau, \Phi)$ be a Hamiltonian $(U, \sigma)$-manifold. Let $T$ be any maximal torus of $U$ and $\mathfrak{t}_{+}^{*}$ a (closed) fundamental Weyl chamber in $\mathfrak{t}^{*}$. For any subset $X$ of $M$ we define $\Delta(X)$ to be the intersection $\Phi(X) \cap \mathfrak{t}_{+}^{*}$. A well-known theorem of Kirwan [17] (as generalized by Hilgert, Neeb and Plank [13] and Sjamaar [28]) says that if $M$ is connected and $\Phi$ is proper, then $\Delta(M)$ is a convex polyhedron in $\mathfrak{t}^{*}$. The main result of this paper is a comparable assertion about the Lagrangian submanifold $M^{\tau}$ of $M$.

For this result to hold, the torus $T$ and the chamber $t_{+}^{*}$ need to be chosen in a suitable "position" relative to the involution $\sigma$, namely as follows. As before, let $K$ be the identity component of $U^{\sigma}$ and $\mathfrak{q} \subseteq \mathfrak{u}$ the -1-eigenspace of the involution $\sigma$. Choose a maximal abelian subspace $\mathfrak{a}$ of $\mathfrak{q}$ and a maximal abelian subalgebra $\mathfrak{t}$ of $\mathfrak{u}$ which contains a. Let $A=\exp \mathfrak{a}$ and $T=\exp \mathfrak{t}$ be the corresponding subtori of $U$. Let $R$ be the root system of $(U, T), R^{\prime} \subseteq R$ the subsystem of roots which vanish on $\mathfrak{a}$, and $R^{\mathfrak{a}}$ the restricted root system. (See Appendix B.) Fix sets of positive roots $R_{+}^{\prime}$ in $R^{\prime}$ and $R_{+}^{\mathfrak{a}}$ in $R^{\mathfrak{a}}$. Define the set of positive roots $R_{+}$in $R$ to be the union of $R_{+}^{\prime}$ and those roots in $R$ which restrict to elements of $R_{+}^{\mathfrak{a}}$. Denote the 
corresponding fundamental Weyl chambers in $\mathfrak{t}^{*}$ and $\mathfrak{a}^{*}$ by $\mathfrak{a}_{+}^{*}$ and $\mathfrak{t}_{+}^{*}$, respectively. Then by Lemma B.2, $\mathfrak{t}_{+}^{*} \cap \mathfrak{q}^{*}=\mathfrak{t}_{+}^{*} \cap \mathfrak{a}^{*}$ is equal to $\mathfrak{a}_{+}^{*}$.

Now consider the $K$-stable subset $\Phi\left(M^{\tau}\right)$ of $\mathfrak{u}^{*}$ and the intersection

$$
\Delta\left(M^{\tau}\right)=\Phi\left(M^{\tau}\right) \cap \mathfrak{t}_{+}^{*} .
$$

Since $\Phi\left(M^{\tau}\right) \subseteq \mathfrak{q}^{*}, \Delta\left(M^{\tau}\right)$ is equal to $\Phi\left(M^{\tau}\right) \cap \mathfrak{a}_{+}^{*}$. Our main result is the following convexity theorem for $M^{\tau}$.

3.1. Theorem. Assume that $M$ is connected, $M^{\tau}$ is nonempty, and the moment map $\Phi$ is proper.

(i) $\Delta\left(M^{\tau}\right)$ is equal to $\Delta(M) \cap \mathfrak{a}^{*}$ and is therefore a closed convex polyhedral subset of $\mathfrak{a}^{*}$.

(ii) If $\lambda \in \mathfrak{a}_{+}^{*}$ is a vertex of $\Delta\left(M^{\tau}\right)$ and $m \in \Phi^{-1}(\lambda) \cap M^{\tau}$, then $A \subseteq\left[U_{\lambda}, U_{\lambda}\right] U_{m}$. In particular, the vertices of $\Delta\left(M^{\tau}\right)$ which are contained in the relative interior of $\mathfrak{t}_{+}^{*}$ are images under $\Phi$ of $A$-fixed points in $M^{\tau}$.

We call $\Delta\left(M^{\tau}\right)$ the moment polyhedron of $M^{\tau}$. From (1) it follows easily that $\Phi\left(M^{\tau}\right)=\Phi(M) \cap \mathfrak{q}^{*}$. Also, (i]) can be interpreted as saying that if a complete list of inequalities for the polyhedron $\Delta(M)$ is known, then the inequalities for $\Delta\left(M^{\tau}\right)$ can be obtained simply by adding to the list a number of equalities, namely those describing the subspace $\mathfrak{a}^{*}$. However, as we shall see in Section 9, this is an inefficient method for finding the inequalities for $\Delta\left(M^{\tau}\right)$.

In Sections 5 and 6 we prove the theorem for projective and affine varieties. The general case is treated in Section 8, where we reduce it to the algebraic case by means of a local normal form theorem. The algebraic versions of Theorem 3.1 enable us to generalize it to various situations where $M$ is singular (Theorem 5.10 and its corollaries), or where $\Phi$ is not proper (Theorems 6.3 and 9.1). We also prove a result, Theorem 8.2, which describes, for any $m \in M^{\tau}$ mapping into $\Delta\left(M^{\tau}\right)$, the shape of the moment polyhedron near $\Phi(m)$ in terms of the coordinate ring of an affine variety determined by infinitesimal data at $m$.

3.2. Example. If $\sigma$ is the identity map on $U$, then $\mathfrak{a}=\{0\}$. The Lagrangian $M^{\tau}$ is $U$-invariant and satisfies $\Phi\left(M^{\tau}\right)=\{0\}$, so Theorem 3.1 is trivially true.

3.3. Example. If $M$ is compact and $U$ is a torus, Theorem 3.1 says that $\Delta\left(M^{\tau}\right)$ is the convex hull of $\Phi\left(M^{A} \cap M^{\tau}\right)$. If in addition $\sigma(u)=u^{-1}$, then $\Phi\left(M^{\tau}\right)=\Phi(M)$, which is the main result of Duistermaat's paper [6].

3.4. Example. Let $\lambda \in \mathfrak{a}_{+}^{*}=\mathfrak{t}_{+}^{*} \cap \mathfrak{q}^{*}$ and let $M$ be the symmetric coadjoint orbit $U \lambda$ of Example 2.9. Then $M^{\tau}=K \lambda$. Consider the action of the maximal torus $T$ on $M$. By Theorem 3.1, $\Delta\left(M^{\tau}\right)=\operatorname{hull}\left(M^{A} \cap M^{\tau}\right)$. According to Lemma B.4(iii), $M^{A} \cap M^{\tau}$ is equal to $W^{\mathfrak{a}} \lambda$, the restricted Weyl group orbit through $\lambda$. The conclusion is that $\Delta\left(M^{\tau}\right)=$ hull $W^{\mathfrak{a}} \lambda$. This was proved by Kostant in 20]. Here is a slightly different way of recovering the same result: Kostant also proved that $\Delta(M)=$ hull $W \lambda$. Therefore, by Theorem 3.1 and Lemma B.4(iii),

$$
\Delta\left(M^{\tau}\right)=\Delta(M) \cap \mathfrak{a}^{*}=(\text { hull } W \lambda) \cap \mathfrak{a}^{*}=\text { hull } W^{\mathfrak{a}} \lambda .
$$

See Section 9 for yet another proof.

We emphasize that $M^{\tau}$ is not stable under the action of the torus $A$. Duistermaat has given a more intrinsic description of the set $M^{A} \cap M^{\tau}$, which does not refer to 
the action of $A$ on the ambient manifold $M$. The submanifold $M^{\tau}$ is stable under the 2-torsion subgroup $A[2]=A^{\sigma}$, and clearly $M^{A} \cap M^{\tau}$ is included in $\left(M^{\tau}\right)^{A[2]}$. This inclusion is often, but not always, an equality. (For an example where it is not, let $U=\mathbf{S U}(2), \sigma=$ complex conjugation, so that $U^{\sigma}=K=\mathbf{S O}(2)$ and $A=T$. Let $M$ be a coadjoint $U$-orbit. Then $M$ is a sphere, $M^{\tau}$ is a great circle, $M^{A} \cap M^{\tau}=M^{A}$ consists of the two poles, but $A[2]$ is the centre of $U$, so it acts trivially on $M$.) To remedy this situation, define the sequence of submanifolds $Y_{k}$ of $M^{\tau}$ by $Y_{k}=M^{A\left[2^{k}\right]} \cap M^{\tau}$ for $k \geq 0$, where $A\left[2^{k}\right]$ denotes the $2^{k}$-torsion subgroup of $A$. One easily proves that $A\left[2^{k}\right]$ maps $Y_{k-1}$ into itself and thus that $Y_{k}=Y_{k-1}^{A\left[2^{k}\right]}$ for $k \geq 1$. Because $\left\{Y_{k}\right\}$ is a nested sequence of submanifolds, it is eventually constant. Since $\bigcup_{k \geq l} A\left[2^{k}\right]$ is dense in $A$, we obtain $M^{A} \cap M^{\tau}=Y_{l}$ for sufficiently large $l$. (Cf. [6, Lemma 3.3].)

\section{Geometric quantization}

The proof of Theorem 3.1 in the algebraic case makes use of geometric quantization. Given a Hamiltonian $(U, \sigma)$-manifold $M$ which admits a Kähler polarization, how is the involution on $M$ reflected in the quantization $Q(M)$ ? In this section we show that, subject to a compatibility condition on the involution and the polarization, $Q(M)$ is a unitary $(U, \sigma)$-module as in Example 2.7. The compatibility condition is for example satisfied for a class of coadjoint orbits which contains, but in some cases is strictly larger than, the class of symmetric coadjoint orbits. For such an orbit the quantization is a unitary $(U, \sigma)$-module which is irreducible as a $U$-module. As a byproduct we obtain a characterization in terms of the orbit method of representations of real and quaternionic type, and a classification of unitary $(U, \sigma)$-modules.

The details are as follows. Let $(M, \omega, \tau, \Phi)$ be a Hamiltonian $(U, \sigma)$-manifold. Suppose that the symplectic form is integral. Let $L$ be a prequantum line bundle, that is a complex line bundle with Hermitian fibre metric $h$ and Hermitian connection $\nabla$ with curvature $-2 \pi i \omega$.

4.1. Definition. The prequantum bundle $L$ is $(U, \sigma)$-equivariant if there exist a lifting of the $U$-action on $M$ to a unitary action on $L$ and a lifting of $\tau$ to a real linear bundle map $\tau_{L}: L \rightarrow L$ which is conjugate linear $\left(\tau_{L}(c l)=\bar{c} \tau_{L}(l)\right.$ for all $l \in L$ and $c \in \mathbb{C}$ ), maps horizontal subspaces to horizontal subspaces, and is an involution $\left(\tau_{L}^{2}=\operatorname{id}_{L}\right)$.

The lifting $\tau_{L}$ defines an isomorphism $(L, \nabla) \rightarrow(\bar{L}, \bar{\nabla})$ of complex line bundles with connection and therefore induces an an equivalence $\left(\tau^{*} L, \tau^{*} \nabla\right) \cong(\bar{L}, \bar{\nabla})$.

The vector fields generating the $U$-actions on $M$ and $L$ are related by Kostant's formula

$$
\xi_{L}=\left(\xi_{M}\right)_{\text {hor }}+2 \pi \Phi^{\xi} \nu_{L}
$$

for $\xi \in \mathfrak{u}$. (See e.g. 10, 19.) Here "hor" refers to the horizontal lift of a vector field and $\nu_{L}$ denotes the infinitesimal generator of the scalar $S^{1}$-action on $L$. This shows that the obstruction to lifting the $U$-action is purely topological and that the lift is unique, if it exists.

Let us now analyse the condition on $\tau$. Because $\tau$ is antisymplectic, the curvature of $\left(\tau^{*} L, \tau^{*} \nabla\right)$ is equal to that of $(\bar{L}, \bar{\nabla})$, and therefore $L^{\prime}=\tau^{*} L \otimes \bar{L}^{*}$ is a bundle 
with flat connection $\nabla^{\prime}=\tau^{*} \nabla \otimes 1+1 \otimes \bar{\nabla}^{*}$. Hence, according to the exact sequence

$$
H^{1}(M, \mathbb{Z}) \hookrightarrow H^{1}(M, \mathbb{R}) \longrightarrow H^{1}\left(M, S^{1}\right) \longrightarrow H^{2}(M, \mathbb{Z}) \longrightarrow H^{2}(M, \mathbb{R}) \longrightarrow \cdots,
$$

the pair $\left(L^{\prime}, \nabla^{\prime}\right)$ is represented up to equivalence by a class in $H^{1}\left(M, S^{1}\right)$. We conclude that a connection preserving conjugate linear lifting $\tau_{L}$ of $\tau$ exists if and only if this class vanishes. According to Proposition 1.12.2 of Kostant [19], any another such lifting $\tau_{L}^{\prime}$ is given by $\tau_{L}^{\prime}=c \tau_{L}$ for some nonzero complex number $c$. Moreover, we can choose $c$ such that $\tau_{L}^{\prime}$ maps $h$ to $\bar{h}$, that is to say, preserves the real inner product Re $h$. Such a lifting is "almost" an involution.

4.2. Lemma. Let $\tau_{L}$ be conjugate linear lifting of $\tau$ which preserves the connection.

(i) If $\tau_{L}$ preserves $\operatorname{Re} h$, then $\tau_{L}^{2}=a \mathrm{id}_{L}$ for some complex number a of norm 1 .

(ii) If $\tau_{L}$ is an involution, then $\tau_{L}$ preserves $\operatorname{Re} h$.

(iii) Let $\tau_{L}^{\prime}$ be another conjugate linear lifting of $\tau$ which preserves the connection. If $\tau_{L}$ is an involution, then $\tau_{L}^{\prime}$ is an involution if and only if it is of the form $\tau_{L}^{\prime}=c \tau_{L}$ with $|c|=1$.

Proof. Assume that $\tau_{L}$ preserves Re $h$. Since $\tau$ is an involution, the map $\tau_{L}^{2}$ is then a (complex linear) automorphism of $L$ which covers the identity and preserves $\nabla$ and $h$. It follows from the aforementioned result of Kostant's that $\tau_{L}^{2}=a \mathrm{id}_{L}$ for some $a$ in the unit circle. This proves (i).

Again by Kostant's result, the fact that $\tau_{L}$ is conjugate linear implies that $\tau_{L}^{*} h=$ $b \bar{h}$ for some $b>0$, and hence $\tau_{L}^{*} \operatorname{Re} h=b \operatorname{Re} h$. If $\tau_{L}$ is an involution, then $\operatorname{Re} h=$ $\left(\tau_{L}^{*}\right)^{2} \operatorname{Re} h=b^{2} \operatorname{Re} h$, so $b=1$ and $\tau_{L}^{*} \operatorname{Re} h=\operatorname{Re} h$. This proves (闬).

Finally, assume that $\tau_{L}$ is an involution. We know that $\tau_{L}^{\prime}=c \tau_{L}$ for some $c \neq 0$. Therefore $\tau_{L}^{\prime}$ is an involution if and only if $\operatorname{id}_{L}=\left(\tau_{L}^{\prime}\right)^{2}=c \tau_{L} c \tau_{L}=c \bar{c} \tau_{L}^{2}=|c|^{2} \mathrm{id}_{L}$, i.e. $|c|=1$. This proves (iii).

4.3. Remark. Even if $\tau$ is liftable, it is not always possible to find an involutive lift. In some cases one can only find a lift $\tau_{L}$ which satisfies the "quaternionic" condition $\tau_{L}^{2}(l)=-l$. See also Remark 4.9.

Suppose from now on that $M$ admits an integrable complex structure $J$ which is $U$-invariant, $\tau$-anti-invariant and compatible with $\omega$. Then $\omega$ is a positive $(1,1)$ form, so $L$ admits a holomorphic structure such that the projection $L \rightarrow M$ is holomorphic. In addition, we can choose the metric connection $(\nabla, h)$ to be compatible with the holomorphic structure.

4.4. Lemma. Let $\tau_{L}: L \rightarrow L$ be any real linear bundle map which lifts $\tau$ and preserves the real inner product $\operatorname{Re} h$. Then $\tau_{L}$ is antiholomorphic if and only if $\tau_{L}$ is conjugate linear and preserves the connection.

Proof. Assume that $\tau_{L}$ is antiholomorphic. Then the derivative of $\tau_{L}$ at any point in $L$ is a conjugate linear map. By taking a point $m$ in the zero section we see that the restriction of $\left(\tau_{L}\right)_{*}$ to $L_{m}$ is a conjugate linear map $L_{m} \rightarrow L_{\tau(m)}$, which implies that the restriction of $\tau_{L}$ to every fibre is conjugate linear. Let $f: L \rightarrow \mathbb{R}$ be the smooth function defined by $f(l)=h(l, l)$. The fact that the connection is Hermitian implies that the horizontal subspace at $l$ is equal to

$$
\mathcal{H}_{l}=\operatorname{ker} d_{l} f \cap i \operatorname{ker} d_{l} f \text {. }
$$


Together with the fact that $\tau_{L}$ is antiholomorphic and preserves $f$ this implies that $\left(\tau_{L}\right)_{*} \mathcal{H}_{l}=\mathcal{H}_{\tau_{L}(l)}$ for all $l \in L$, that is to say $\tau_{L}$ preserves the connection.

Now assume that $\tau_{L}$ is conjugate linear and preserves the connection. The tangent space to $L$ at any point $l$ decomposes as $T_{l} L=\mathcal{H}_{l} \oplus L_{m}$, where $m$ is the base point of $l$. Because $\tau_{L}$ preserves the connection, its derivative at $l$ is the direct sum of two maps $L_{m} \rightarrow L_{\tau(m)}$ and $\mathcal{H}_{l} \rightarrow \mathcal{H}_{\tau_{L}(l)}$. Both of these maps are conjugate linear, the first one by assumption and the second one because $\tau$ is antiholomorphic and the connection is complex. Therefore $\left(\tau_{L}\right)_{*}$ is conjugate linear, i.e. $\tau_{L}$ is antiholomorphic.

Henceforth we assume $L$ to be $(U, \sigma)$-equivariant and fix an involutive lifting $\tau_{L}$ as in Definition 4.1. Then $\tau_{L}$ preserves Re $h$ by Lemma 4.2 and hence is antiholomorphic by Lemma 4.4. Let $Q(M)=\Gamma(M, L)$ be the space of global holomorphic sections of $L$. We define an involution on the global smooth sections of $L$ by sending a section $s$ to $\tau_{L} \circ s \circ \tau$. Because $\tau$ and $\tau_{L}$ are both antiholomorphic, this involution maps $Q(M)$ into itself. We denote its restriction to $Q(M)$ by $\tau_{Q}$. Since $\tau_{L}$ is conjugate linear, so is $\tau_{Q}$. Moreover, $\tau_{Q}$ preserves the real part of the Hermitian inner product on $Q(M)$ given by $\langle s, t\rangle=\int_{M} h(s(m), t(m)) d m$, where $d m=\left|\omega^{n} / n !\right|$ is Liouville measure on $M$. With this inner product, $Q(M)$ is also a unitary $U$-module in a natural way. The involution and the $U$-representation on $Q(M)$ are related in the expected manner. Since $\tau_{L}$ preserves the connection, we have $\left(\tau_{L}\right)_{*} X_{\text {hor }}=\left(\tau_{*} X\right)_{\text {hor }}$ for all tangent vectors $X$. Together with (2.1), (2.3) and (1.1) this implies that for all $\xi \in \mathfrak{u}$

$$
\begin{aligned}
\left(\tau_{L}\right)_{*} \xi_{L} & =\left(\tau_{L}\right)_{*}\left(\left(\xi_{M}\right)_{\mathrm{hor}}\right)+2 \pi\left(\Phi^{\xi} \circ \tau\right)\left(\tau_{L}\right)_{*} \nu_{L} \\
& =\left(\tau_{*} \xi_{M}\right)_{\mathrm{hor}}-2 \pi \Phi^{\sigma(\xi)}\left(-\nu_{L}\right) \\
& =\left(\sigma(\xi)_{M}\right)_{\mathrm{hor}}+2 \pi \Phi^{\sigma(\xi)} \nu_{L} \\
& =\sigma(\xi)_{L} .
\end{aligned}
$$

Therefore $\tau_{L}(u l)=\sigma(u) \tau_{L}(l)$ for all $u \in U$ and $l \in L$. From this we obtain easily that $\tau_{Q}(u s)=\sigma(u) \tau_{Q}(s)$ for all $u \in U$ and holomorphic sections $s$. The result is as follows.

4.5. Proposition. The vector space $Q(M)$ is a unitary $(U, \sigma)$-module as in Example 2.7, finite-dimensional if $M$ is compact. Two different involutive connectionpreserving liftings of $\tau$ give rise to isomorphic unitary $(U, \sigma)$-modules.

Proof. It remains to prove the uniqueness part. According to Lemma 4.2, any other lifting $\tau_{L}^{\prime}$ with the stated properties is of the form $\tau_{L}^{\prime}=c \tau_{L}$ with $|c|=1$. Then $\tau_{L}^{\prime}$ gives rise to the involution $\tau_{Q}^{\prime}=c \tau_{Q}$ of $Q(M)$. The scalar multiplication operator $C(s)=\sqrt{c} s$ is unitary, commutes with $U$ and satisfies $C \tau_{Q} C^{-1}=C^{2} \tau_{Q}=\tau_{Q}^{\prime}$, so defines a unitary intertwining operator between the $(U, \sigma)$-modules $\left(Q(M), \tau_{Q}\right)$ and $\left(Q(M), \tau_{Q}^{\prime}\right)$.

Kodaira's Embedding Theorem implies that if $M$ is compact, it is biholomorphically equivalent to a complex projective variety. Using Proposition 4.5 one can easily show that Kodaira's map, $j$, is $U \rtimes\{ \pm 1\}$-equivariant, and hence that its image $j(M)$ is a $U$-stable and $\tau$-stable nonsingular projective variety, as in Example 2.11. In general $j$ is not a symplectomorphism, but a straightforward Darboux-type argument shows that there exists a $U \rtimes\{ \pm 1\}$-equivariant diffeomorphism $f$ from $M$ to itself such that $j \circ f$ is a symplectomorphism. The conclusion is as follows. 
4.6. Corollary. Every compact Hamiltonian $(U, \sigma)$-manifold admitting a $(U, \sigma)$ equivariant Kähler prequantization is symplectically and $(U, \sigma)$-equivariantly diffeomorphic to a complex projective $(U, \sigma)$-manifold.

Using Proposition 4.5 we can give a complete description of all finite-dimensional $(U, \sigma)$-modules. Let us first investigate the irreducible case. It is well-known that the Kähler quantization of a Hamiltonian $U$-manifold is an irreducible $U$-module if and only if the manifold is isomorphic to an integral coadjoint orbit, i.e. an orbit of the form $U \lambda$ with $\lambda$ integral dominant. Such an orbit is diffeomorpic in a natural way to the complex homogeneous space $U^{\mathbb{C}} / P_{\lambda}$, where $U^{\mathbb{C}}$ is the complexification of $U$ and $P_{\lambda}$ is the parabolic subgroup associated with $\lambda$. It follows from the Borel-Weil Theorem that the quantization of $U \lambda$ is the irreducible module $V_{\lambda}$ with highest weight $\lambda$. We shall establish a necessary and sufficient criterion for $V_{\lambda}$ to be a $(U, \sigma)$-module.

First we need an auxiliary result. Let $K^{\prime}$ be the centralizer of $A$ in $K$. Then $T^{\prime}=\left(K^{\prime}\right)_{0} \cap T$ is a maximal torus of $K^{\prime}$. Let $W^{\prime} \subseteq W$ the Weyl group of (the identity component of) $K^{\prime}$ with respect to $T^{\prime}$ and let $w_{0}^{\prime}$ be the longest element of $W^{\prime}$. Define $\sigma_{+}: \mathfrak{t}^{*} \rightarrow \mathfrak{t}^{*}$ by $\sigma_{+}(\lambda)=-\sigma\left(w_{0}^{\prime} \lambda\right)$. As before, $R_{+}$denotes a set of positive roots in $\mathfrak{t}^{*}$ chosen as in (B.4).

4.7. Lemma. (i) $\sigma_{+}$is an involution and $\sigma_{+}\left(R_{+}\right)=R_{+}$. Hence $\sigma_{+}\left(\mathfrak{t}_{+}^{*}\right)=\mathfrak{t}_{+}^{*}$ and $\sigma_{+}$preserves the partial ordering on the weight lattice defined by $R_{+}$.

(ii) $\sigma_{+}(\lambda)=\lambda$ if and only if either $\lambda \in \mathfrak{a}^{*}$, or $\lambda \in\left(\mathfrak{t}^{\prime}\right)^{*}$ and $\lambda=-w_{0}^{\prime} \lambda$.

(iii) Let $k_{0} \in \mathcal{N}_{\left(K^{\prime}\right)_{0}}\left(T^{\prime}\right)$ be any representative of $w_{0}^{\prime}$. Let $\lambda$ be an integral weight and let $\chi_{\lambda}$ denote the character of $T$ obtained by exponentiating $\lambda$. If $\lambda \in \mathfrak{a}^{*}$, then $\chi_{\lambda}\left(k_{0}^{2}\right)=1$. If $\lambda \in\left(\mathfrak{t}^{\prime}\right)^{*}$ and $\lambda=-w_{0}^{\prime} \lambda$, then $\chi_{\lambda}\left(k_{0}^{2}\right)= \pm 1$.

Proof. The action of $W^{\prime}$ fixes $\mathfrak{a}^{*}$ and therefore commutes with $\sigma$. Since $w_{0}^{\prime}$ and $\sigma$ are involutions, so is $\sigma_{+}$. Observe that $w_{0}^{\prime}$ maps $R_{+}^{\prime}$ to $R_{-}^{\prime}$ and permutes $R_{+} \backslash R_{+}^{\prime}$, whereas $\sigma$ fixes $R_{+}^{\prime}$ and sends $R_{+} \backslash R_{+}^{\prime}$ to $R_{-} \backslash R_{-}^{\prime}$. This implies that $w_{0}^{\prime} \circ \sigma$ maps $R_{+}$to $R_{-}$. Therefore $\sigma_{+}\left(R_{+}\right)=R_{+}$. This proves (i).

The fact that $w_{0}^{\prime}$ fixes $\mathfrak{a}^{*}$ implies that $\sigma_{+}$respects the decomposition (B.3), and that $\left.\sigma_{+}\right|_{\mathfrak{a}^{*}}=\mathrm{id}$ and $\left.\sigma_{+}\right|_{\left(\mathfrak{t}^{\prime}\right)^{*}}=-w_{0}^{\prime}$. This immediately implies (ii).

Let $\lambda$ be an integral weight. Since $w_{0}^{\prime}$ is an involution, $k_{0}^{2}$ is in $T^{\prime}$. If $\lambda \in \mathfrak{a}^{*}$, then $T^{\prime}$ is contained in the kernel of $\chi_{\lambda}$, so $\chi_{\lambda}\left(k_{0}^{2}\right)=1$. Now suppose $\lambda \in\left(\mathfrak{t}^{\prime}\right)^{*}$ satisfies $\lambda=-w_{0}^{\prime} \lambda$. Then $\chi_{\lambda}\left(k_{0} t k_{0}^{-1}\right)=\chi_{\lambda}(t)^{-1}$ for all $t \in T$. Taking $t=k_{0}^{2}$ we obtain $\chi_{\lambda}\left(k_{0}^{2}\right)=\chi_{\lambda}\left(k_{0}^{2}\right)^{-1}$.

4.8. Theorem. Let $\lambda$ be an integral dominant weight of $U$. Then the irreducible $U$-module $V_{\lambda}$ is a $(U, \sigma)$-module if and only if $\sigma_{+}(\lambda)=\lambda$ and $\chi_{\lambda}\left(k_{0}^{2}\right)=1$, where $k_{0}$ is as in Lemma 4.7(iii).

In particular, $V_{\lambda}$ is a $(U, \sigma)$-module if $\lambda \in \mathfrak{a}^{*}$. It is easy to check that if $\sigma_{+}(\lambda)=\lambda$ the condition $\chi_{\lambda}\left(k_{0}^{2}\right)=1$ does not depend on the choice of the representative $k_{0}$ of $w_{0}^{\prime}$.

Proof. Let $(V, \tau)$ be any $(U, \sigma)$-module and let $v$ be a weight vector in $V$ with weight $\mu$. The rule $\tau(u v)=\sigma(u) \tau(v)$ implies that $\tau(v)$ is a weight vector with weight $-\sigma(\mu)$, and therefore

$$
k_{0} \tau(v) \text { is a weight vector with weight } \sigma_{+}(\mu) .
$$


Now consider the orbit $M=U \lambda$ and the $U$-module $Q(M)=V_{\lambda}$. Assume that $V_{\lambda}$ is a $(U, \sigma)$-module. Let $v$ be a highest-weight vector. Then $k_{0} \tau(v)$ has weight $\sigma_{+}(\lambda)$ according to (4.3). We conclude from Lemma 4.7(日) that $\sigma_{+}(\lambda)=\lambda$ and $k_{0} \tau(v)=c v$ for some $c \in \mathbb{C}$. This implies $\left(k_{0} \tau\right)^{2}(v)=|c|^{2} v$ because $\tau$ is conjugate linear. Hence

$$
\chi_{\lambda}\left(k_{0}^{2}\right) v=k_{0}^{2} v=k_{0}^{2} \tau^{2}(v)=\left(k_{0} \tau\right)^{2}(v)=|c|^{2} v,
$$

where we have used that $\tau$ commutes with $k_{0}$ since $k_{0} \in K$. This implies $\chi_{\lambda}\left(k_{0}^{2}\right)=1$, since $\left|\chi_{\lambda}\left(k_{0}^{2}\right)\right|=1$.

Now assume that $\sigma_{+}(\lambda)=\lambda$ and $\chi_{\lambda}\left(k_{0}^{2}\right)=1$. Then $-\sigma(\lambda)=w_{0}^{\prime} \lambda$, so by Example 2.9 the orbit $M$ has a well-defined antisymplectic involution $\tau=-\sigma$. Let us check that $\tau$ is antiholomorphic. Because the $U$-action is holomorphic and transitive, we need only check that $\left(\mathcal{L}_{k_{0}}\right)_{*} \circ \tau_{*}: T_{\lambda} M \rightarrow T_{\lambda} M$ is conjugate linear, where " $\mathcal{L}$ " denotes left multiplication on $M$ by elements of $U$. Since $w_{0}^{\prime}$ is an involution,

$$
\tau(u \lambda)=\sigma(u) \tau(\lambda)=\sigma(u) w_{0}^{\prime} \lambda=\sigma(u) k_{0}^{-1} \lambda .
$$

Let us identify $T_{\lambda} M$ with $\mathfrak{u} / \mathfrak{u}_{\lambda}$. Left-multiplying by $k_{0}$ and differentiating at 1 we obtain from (4.4) the commutative diagram

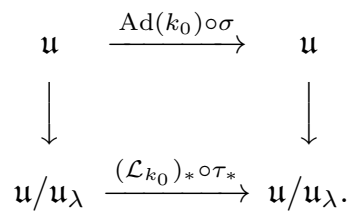

Consider the root-space decomposition $\mathfrak{u}^{\mathbb{C}}=\mathfrak{t}^{\mathbb{C}} \oplus \bigoplus_{\alpha \in R} \mathfrak{u}_{\alpha}^{\mathbb{C}}$ and select root vectors $E_{\alpha} \in \mathfrak{u}_{\alpha}^{\mathbb{C}} \backslash\{0\}$ such that $E_{-\alpha}=-\bar{E}_{\alpha}$ for all $\alpha \in R$. Denote the complex linear extension of $\operatorname{Ad}\left(k_{0}\right) \circ \sigma$ by $C$. Observe that $C \mathfrak{u}_{\alpha}^{\mathbb{C}}=\mathfrak{u}_{-\sigma_{+}(\alpha)}^{\mathbb{C}}$, which implies that there exist $c_{\alpha} \in \mathbb{C}$ such that

$$
C E_{\alpha}=c_{\alpha} E_{-\sigma_{+}(\alpha)} \quad \text { and } \quad C E_{-\alpha}=\bar{c}_{\alpha} E_{\sigma_{+}(\alpha)}
$$

for $\alpha \in R_{+}$. Let $R^{\lambda}=R_{+}^{\lambda} \cup R_{-}^{\lambda}$ be the set of all roots which annihilate $\lambda$. That is to say, $\alpha \in R^{\lambda}$ if and only $\alpha^{\vee}(\lambda)=0$, where $\alpha^{\vee}$ denotes the dual root of $\alpha$. Then $\sigma_{+}\left(R_{ \pm}^{\lambda}\right)=R_{ \pm}^{\lambda}$ because $\sigma_{+}(\lambda)=\lambda$ and $\sigma_{+}$maps $R_{+}$into itself. Hence the Lie algebra of the parabolic subgroup $P_{\lambda}$ is given by

$$
\mathfrak{p}_{\lambda}=\mathfrak{t}^{\mathbb{C}} \oplus \bigoplus_{\alpha \in R_{+}} \mathfrak{u}_{\alpha}^{\mathbb{C}} \oplus \bigoplus_{\alpha \in R_{-}^{\lambda}} \mathfrak{u}_{\alpha}^{\mathbb{C}}
$$

Therefore

$$
\begin{aligned}
\mathfrak{u}^{\mathbb{C}} / \mathfrak{p}_{\lambda} & \cong \bigoplus_{\alpha \in R_{-} \backslash R_{-}^{\lambda}} \mathbb{C} E_{\alpha}, \\
T_{\lambda} M \cong \mathfrak{u} / \mathfrak{u}_{\lambda} & \cong \bigoplus_{\alpha \in R_{-} \backslash R_{-}^{\lambda}}\left(\mathbb{R} X_{\alpha} \oplus \mathbb{R} Y_{\alpha}\right),
\end{aligned}
$$

where $X_{\alpha}=\frac{1}{2 i}\left(E_{-\alpha}+E_{\alpha}\right)$ and $Y_{\alpha}=\frac{1}{2}\left(E_{-\alpha}-E_{\alpha}\right)$. The complex structure on $T_{\lambda} M$ is by definition the pullback of the complex structure on $\mathfrak{u}^{\mathbb{C}} / \mathfrak{p}_{\lambda}$, so we obtain from (4.7) that $J X_{\alpha}=Y_{\alpha}$ and $J Y_{\alpha}=-X_{\alpha}$. It follows from (4.6) that for all 
$\alpha \in R_{-} \backslash R_{-}^{\lambda}$

$$
\begin{aligned}
C X_{\alpha} & =a_{\alpha} X_{\sigma_{+}(\alpha)}+b_{\alpha} Y_{\sigma_{+}(\alpha)}, \\
C Y_{\alpha} & =b_{\alpha} X_{\sigma_{+}(\alpha)}-a_{\alpha} Y_{\sigma_{+}(\alpha)},
\end{aligned}
$$

where $a_{\alpha}=\operatorname{Re} c_{\alpha}$ and $b_{\alpha}=\operatorname{Im} c_{\alpha}$. Thus $C J=-J C$. Together with (4.5) this proves that $\left(\mathcal{L}_{k_{0}}\right)_{*} \circ \tau_{*}$ is conjugate linear on $\mathfrak{u} / \mathfrak{u}_{\lambda}$.

Next we show that $U \lambda$ possesses a $(U, \sigma)$-equivariant prequantum line bundle. Let $L$ be the homogeneous bundle

$$
L=U \times{ }^{U_{\lambda}} \mathbb{C}_{\lambda} \cong U^{\mathbb{C}} \times{ }^{P_{\lambda}} \mathbb{C}_{\lambda},
$$

where $\mathbb{C}_{\lambda}$ denotes the obvious one-dimensional representation of $U_{\lambda}$, resp. $P_{\lambda}$. This bundle has a $U$-invariant Hermitian structure, namely the one induced by the standard inner product on $\mathbb{C}$. Put

$$
\tau_{L}[u, z]=\left[\sigma(u) k_{0}^{-1}, \bar{z}\right] .
$$

The assumption that $\sigma_{+}(\lambda)=\lambda$ implies that $\tau_{L}$ is well-defined as a map from $L$ to itself. The assumption that $\chi_{\lambda}\left(k_{0}^{2}\right)=1$ implies that it is an involution. It is clear that $\tau_{L}$ is a conjugate linear bundle map and it follows from (4.4) that it is a lifting of the involution $\tau$ on $M$. The length function on $L$ is given by $f([u, z])=|z|^{2}$, which is evidently preserved by $\tau_{L}$. One checks directly that $\tau_{L}(u l)=\sigma(u) \tau_{L}(l)$, so $\left(\tau_{L}\right)_{*} \xi_{L}=\sigma(\xi)_{L}$ for all $\xi \in \mathfrak{u}$. Furthermore, $\left(\tau_{L}\right)_{*} \nu_{L}=-\nu_{L}$ and $\tau^{*} \Phi^{\xi}=-\Phi^{\sigma(\xi)}$, so by reading (4.2) backwards we get $\left(\tau_{L}\right)_{*}\left(\left(\xi_{M}\right)_{\text {hor }}\right)=\left(\tau_{*} \xi_{M}\right)_{\text {hor }}$ for all $\xi$. Since $M$ is homogeneous, this implies $\left(\tau_{L}\right)_{*} \mathcal{H}_{l}=\mathcal{H}_{\tau_{L}(l)}$ for all $l \in L$, and so $\tau_{L}$ preserves the connection. Therefore $\tau_{L}$ is antiholomorphic by Lemma 4.4. The upshot is that $L$ is a $(U, \sigma)$-equivariant prequantum line bundle on $M$. Hence, by Proposition 4.5, $V_{\lambda}=Q(M)$ is a unitary $(U, \sigma)$-module.

4.9. Remark (quaternionic modules). If $\sigma_{+}(\lambda)=\lambda$ but $\chi_{\lambda}\left(k_{0}^{2}\right)=-1$, then the lifting $\tau_{L}$ is not an involution, but satisfies $\tau_{L}^{2}[u, z]=[u,-z]$. Otherwise the proof goes through as stated and the result is a unitary irreducible $U$-module $Q(M)$ with an antiholomorphic orthogonal map $\tau_{Q}$ satisfying $\tau_{Q}^{2}=-\mathrm{id}$ and $\tau_{Q}(u v)=\sigma(u) v$. In the extreme case where $\sigma$ is the identity such a module is known as a $U$-module of quaternionic (or symplectic) type, and what we have called a $(U, \sigma)$-module is nothing but a $U$-module of real (or orthogonal) type. The condition $\sigma_{+}(\lambda)=\lambda$ is then equivalent to $\lambda=-w_{0} \lambda$ ( $w_{0}$ being the longest element in $W$ ), in other words to the representation $V_{\lambda}$ being self-conjugate.

4.10. Example. If $U=\mathbf{S U}(n)$ and $\sigma$ is complex conjugation, then $U^{\sigma}=K=$ $\mathbf{S O}(n)$ and $\mathfrak{a}=\mathfrak{t}$. Hence every coadjoint orbit is symmetric and every irreducible $U$ module admits the structure of a $(U, \sigma)$-module. On the fundamental representation $\Lambda^{k} \mathbb{C}^{n}$ the involution is simply complex conjugation with respect to a real form $\bigwedge^{k} \mathbb{R}^{n}$. On the other hand, if $\sigma$ is the identity, then $\mathfrak{a}=\{0\}$ and only a few orbits give rise to representations of real type. For instance, $\Lambda^{k} \mathbb{C}^{n}$ is self-dual if and only if $n$ is even and $k=n / 2$. It is of real type if and only if $n \equiv 0 \bmod 4$ and $k=n / 2$. (See e.g. Tits [30].)

We can now easily describe the structure of an arbitrary $(U, \sigma)$-module.

4.11. Addendum. (i) Every finite-dimensional object in the category of $(U, \sigma)$ modules is isomorphic to a direct sum of irreducible objects. 
(ii) The irreducible objects are

$$
\begin{array}{ll}
V_{\lambda} & \text { with } \sigma_{+}(\lambda)=\lambda \text { and } \chi_{\lambda}\left(k_{0}^{2}\right)=1, \\
V_{\lambda}^{\text {dup }} & \text { with } \sigma_{+}(\lambda) \neq \lambda \text { or } \chi_{\lambda}\left(k_{0}^{2}\right)=-1 .
\end{array}
$$

Here $\lambda$ ranges over the dominant weights of $U$ and $V_{\lambda}^{\text {dup }}$ stands for the duplicated module defined in Example 2.15.

(iii) As a $U$-module $V_{\lambda}^{\text {dup }}$ is isomorphic to $V_{\lambda} \oplus V_{\sigma_{+}(\lambda)}$.

Note that a module of type (4.9) can be described as the quantization of the disconnected manifold $(U \lambda)^{\text {dup }} \cong U \lambda \sqcup-\sigma(U \lambda)$.

Proof. (i) is proved by induction on the dimension and (iii) follows from (4.3) and Lemma 4.7(保).

It is clear that the objects (4.8) are irreducible and it follows from Theorem 4.8 that the objects $(4.9)$ are irreducible as well. Now let $(V, \tau)$ be an arbitrary irreducible object. If it is irreducible as a $U$-module, we conclude from Theorem 4.8 that it must be of type (4.8). If not, it must be of the form $V_{\lambda} \oplus \tau\left(V_{\lambda}\right) \cong$ $V_{\lambda} \oplus V_{\sigma_{+}(\lambda)} \cong V_{\lambda}^{\text {dup }}$ for some $\lambda \in \Lambda_{+}^{*}$. In this case we cannot have $\sigma_{+}(\lambda)=\lambda$ and $\chi_{\lambda}\left(k_{0}^{2}\right)=1$, for then $V_{\lambda}^{\text {dup }}$ would be isomorphic to $V_{\lambda} \oplus V_{\lambda}$ as a $(U, \sigma)$-module. (The easiest way to see this is by using the bilinear form $b$ defined in (2.5). It is not hard to show that if a $U$-module $E$ carries two nondegenerate symmetric bilinear forms $b_{1}, b_{2}$ satisfying $b_{i}(u v, \sigma(u) w)=b_{i}(v, w)$, then $f^{*} b_{1}=b_{2}$ for some $U$-equivariant linear map $f$. Applying this observation to $E=V_{\lambda}^{\text {dup }}$ with $b_{1}$ being the form defined by the involution $\tau$ and $b_{2}$ the one defined by the involutions on each of the summands $V_{\lambda}$, we obtain $V_{\lambda}^{\text {dup }} \cong V_{\lambda} \oplus V_{\lambda}$ as a $(U, \sigma)$-module.) Thus we are in case (4.9). This proves (iii).

4.12. Remark. If $U$ is not connected, (ii) is still true, but the description of the irreducible objects is less clear-cut. However, the fact remains that each such object is either of the form $V$ or $V^{\text {dup }}$, where $V$ is irreducible as a $U$-module.

\section{The projective Case}

In this section we prove Theorem 3.1 for compact integral Kähler manifolds. The gist of the argument is that the Lagrangian $M^{\tau}$ is dense in $M$ for the complex Zariski topology. Noteworthy is that the proof applies to singular as well as nonsingular varieties, and that it leads to a stronger conclusion, Corollaries 5.12 and 5.13, which involve the dual symmetric pair $(G, K)$. On top of this we obtain a simple characterization of the polytope $\Delta\left(M^{\tau}\right)$ in terms of certain rational weights associated with the quantization of $M$, analogous to the characterization of $\Delta(M)$ given by Guillemin and Sternberg [9] and Mumford [27].

Let $(M, \omega, \tau, \Phi)$ be a compact connected Hamiltonian $(U, \sigma)$-manifold equipped with a complex structure which is compatible with $\omega$ and relative to which $\tau$ is antiholomorphic. Furthermore, as in Proposition 4.5, let $L$ be a holomorphic line bundle with a Hermitian connection $\nabla$ such that $\operatorname{curv} \nabla=-2 \pi i \omega$ and $\tau$ lifts to an involutive antiholomorphic bundle map $\tau_{L}$ which preserves $\nabla$.

According to Corollary 4.6 we can alternatively think of $M$ as a $U$-stable and $\tau$-stable complex submanifold of $\mathbb{P} V$ for some unitary $(U, \sigma)$-module $V$.

Following Brion [5], we start by defining a "rational" analogue of the sets $\Delta(M)$ and $\Delta\left(M^{\tau}\right)$. Let $\Lambda_{+}^{*}$ be the monoid of dominant weights in $\mathfrak{t}_{+}^{*}$. 
5.1. Definition. The highest-weight set of $M$ is the subset $\mathcal{C}(M)$ of $\Lambda^{*} \otimes \mathbb{Q}$ consisting of all quotients $\lambda / n$, where $\lambda \in \Lambda_{+}^{*}$ and $n$ is a positive integer such that the irreducible representation $V_{\lambda}$ occurs in the $U$-module $\Gamma\left(M, L^{n}\right)$.

Since

$$
\operatorname{Hom}\left(\Gamma\left(M, L^{n}\right), V_{\lambda}\right) \cong \Gamma\left(M, L^{n}\right) \otimes \Gamma\left(U \lambda^{*}, L_{\lambda^{*}}\right) \cong \Gamma\left(M \times U \lambda^{*}, L^{n} \otimes L_{\lambda^{*}}\right),
$$

we see that $\xi \in \mathcal{C}(M)$ if and only if $\xi=\lambda / n$ for some $\lambda \in \Lambda_{+}^{*}$ and $n>0$ such that there exist $m \in M, u \in U$, and a $U$-invariant global holomorphic section $s$ of $L^{n} \otimes L_{\lambda^{*}}$ with $s\left(m, u \lambda^{*}\right) \neq 0$. Here we use the notation $\lambda^{*}=-w_{0} \lambda$ and the fact that $V_{\lambda}^{*} \cong V_{\lambda^{*}}$.

5.2. Definition. The highest-weight set of $M^{\tau}$ is the set $\mathcal{C}\left(M^{\tau}\right)$ consisting of all quotients $\lambda / n$, with $\lambda \in \Lambda_{+}^{*} \cap \mathfrak{a}^{*}$ and $n>0$, such that there exist $m \in M^{\tau}, k \in K$, and a $U$-invariant global holomorphic section $s$ of $L^{n} \otimes L_{\lambda^{*}}$ with $s\left(m, k \lambda^{*}\right) \neq 0$.

5.3. Lemma. If $M^{\tau}$ is nonempty, then $\mathcal{C}\left(M^{\tau}\right)=\mathcal{C}(M) \cap \mathfrak{a}^{*}$.

Proof. The inclusion $\mathcal{C}\left(M^{\tau}\right) \subseteq \mathcal{C}(M) \cap \mathfrak{a}^{*}$ is obvious. Now let $\xi \in \mathcal{C}(M) \cap \mathfrak{a}^{*}$, write $\xi=\lambda / n$ with $\lambda \in \Lambda_{+}^{*} \cap \mathfrak{a}^{*}$ and $n>0$, and choose a $U$-invariant $s \in \Gamma\left(M \times U \lambda^{*}, L^{n} \otimes\right.$ $\left.L_{\lambda^{*}}\right)$ such that $s\left(m, u \lambda^{*}\right) \neq 0$ for some $m \in M, u \in U$. We need to show that $m$ can be chosen in $M^{\tau}$ and $u$ in $K$.

Let us first consider the case $\xi=0$, where we need merely show that $s(m) \neq 0$ for some $m \in M^{\tau}$. Here the desired result follows from Proposition 2.3, which says that $M^{\tau}$ is a totally real Lagrangian: if $s$ vanished on $M^{\tau}$, then it would vanish on all of $M$ by the identity principle.

The general case is reduced to the special case by means of the "shifting trick": one replaces $M$ with the product $M \times U \lambda^{*}$ and $L$ with $L^{n} \otimes L_{\lambda^{*}}$. Lemma 5.4 below implies that $\lambda^{*} \in \mathfrak{a}^{*}$, so according to Example 2.9 the orbit $U \lambda^{*}$ has a welldefined antisymplectic involution, which we denote here by $\tau_{\lambda^{*}}$, and $\left(U \lambda^{*}\right)^{\tau_{\lambda^{*}}}=$ $K \lambda^{*} \neq \emptyset$ by (2.6). Applying the result of the previous paragraph, we find a point in $M^{\tau} \times\left(U \lambda^{*}\right)^{\tau_{\lambda^{*}}}=M^{\tau} \times K \lambda^{*}$ where $s$ does not vanish, i.e. $m \in M^{\tau}$ and $k \in K$ such that $s\left(m, k \lambda^{*}\right) \neq 0$.

5.4. Lemma. Let $w_{0}^{\mathfrak{a}}$ be the longest element in $W^{\mathfrak{a}}$. Then $w_{0}^{\mathfrak{a}} \lambda=w_{0} \lambda$ for all $\lambda \in \mathfrak{a}^{*}$. It follows that $w_{0} \in W^{\sigma}$.

Proof. Let $\lambda \in \mathfrak{a}_{+}^{*}$. By definition $w_{0}^{\mathfrak{a}}$ satisfies $w_{0}^{\mathfrak{a}} \lambda \in-\mathfrak{a}_{+}^{*} \subseteq-\mathfrak{t}_{+}^{*}$. Furthermore, $w_{0}^{\mathfrak{a}} \lambda=w \lambda$ for some $w \in W^{\sigma}$ by Lemma B.4(1), so $w \lambda \in-\mathfrak{t}_{+}^{*}$. At the same time $w_{0} \lambda \in-\mathfrak{t}_{+}^{*}$ and therefore $w \lambda=w_{0} \lambda$, because $W \lambda$ intersects $-\mathfrak{t}_{+}^{*}$ in a unique point. We conclude that $w_{0}^{\mathfrak{a}} \lambda=w_{0} \lambda$. By Lemma B.4(i) this implies $w_{0} \in \mathcal{N}_{W}(\mathfrak{a})=$ $W^{\sigma}$.

Let $\mathcal{X}(M)$ be the Lie algebra of vector fields on $M$. Since $\mathcal{X}(M)$ has a complex structure, the homomorphism $\mathcal{A}: \mathfrak{u} \rightarrow \mathcal{X}(M)$ which maps $\xi$ to $\xi_{M}$ extends naturally to a homomorphism of complex Lie algebras,

$$
\mathcal{A}^{\mathbb{C}}: \mathfrak{u}^{\mathbb{C}} \longrightarrow \mathcal{X}(M) \text {. }
$$

Recall that the group of biholomorphic transformations of a compact complex manifold is a complex Lie group. It follows from this that $\mathcal{A}^{\mathbb{C}}$ integrates to an action of the complex reductive group $U^{\mathbb{C}}$. Let $\sigma^{\mathbb{C}}$ denote the complex linear extension of $\sigma$ to $\mathfrak{u}^{\mathbb{C}}$ (and also the holomorphic extension of $\sigma$ to $U^{\mathbb{C}}$ ), and let $\gamma_{\mathfrak{u}}$ be complex 
conjugation on $\mathfrak{u}^{\mathbb{C}}$ relative to $\mathfrak{u}$. Put $\gamma_{\mathfrak{g}}=\sigma^{\mathbb{C}} \gamma_{\mathfrak{u}}$. Then $\gamma_{\mathfrak{g}}$ is a conjugate linear involution of $\mathfrak{u}^{\mathbb{C}}$ because $\sigma^{\mathbb{C}} \gamma_{\mathfrak{u}}=\gamma_{\mathfrak{u}} \sigma^{\mathbb{C}}$. It is nothing other than complex conjugation on $\mathfrak{u}^{\mathbb{C}}$ relative to the real form

$$
\mathfrak{g}=\mathfrak{k} \oplus \mathfrak{p},
$$

where $\mathfrak{p}=i \mathfrak{q}$. It follows from (2.2) that $\mathcal{A} \sigma=\tau \mathcal{A}$ and therefore

$$
\mathcal{A}^{\mathbb{C}} \gamma_{\mathfrak{g}}=\tau \mathcal{A}^{\mathbb{C}}
$$

because $\tau$ is conjugate linear. Let us write $G$ for the connected real reductive subgroup of $U^{\mathbb{C}}$ generated by $\exp \mathfrak{g}$ and $\theta$ for the restriction of $\sigma^{\mathbb{C}}$ to $G$. Then $K$ is a maximal compact subgroup of $G, \theta$ is the Cartan involution, and the symmetric pair $(\mathfrak{g}, \mathfrak{k})$ is known as the dual of $(\mathfrak{u}, \mathfrak{k})$; cf. [12, Ch. V]. The following result is analogous to Proposition 2.3.

5.5. Proposition. The involution $\tau$ is G-equivariant. Hence every connected component of $M^{\tau}$ is $G$-stable. For every $m \in M^{\tau}$ the intersection $U^{\mathbb{C}} m \cap M^{\tau}$ has a finite number of connected components, each of which consists of a single G-orbit.

Proof. It follows from (5.2) that $\tau_{*} \xi_{M}=\xi_{M}$ for all $\xi \in \mathfrak{g}$. Consequently $\tau(\mathrm{gm})=$ $g \tau(m)$ for all $g \in G$ and $m \in M$ because $G$ is connected. This implies that $G$ preserves every component of $M^{\tau}$. That $U^{\mathbb{C}} m \cap M^{\tau}$ has finitely many connected components follows from the fact that it is a real algebraic variety. To prove that each component is a single $G$-orbit it suffices to show that the tangent space to $G m$ at $m$ is equal to $T_{m}\left(U^{\mathbb{C}} m\right) \cap T_{m} M^{\tau}$. A general tangent vector to $U^{\mathbb{C}} m$ at $m$ is of the form $\zeta_{M}=\zeta_{M}^{+}+\zeta_{M}^{-}$, where $\zeta^{ \pm}=\xi^{ \pm}+i \eta^{ \pm}$with $\xi^{+}, \eta^{+} \in \mathfrak{k}$ and $\xi^{-}, \eta^{-} \in \mathfrak{q}$. According to $(5.2)$,

$$
\tau_{*}\left(\zeta_{M}\right)=\gamma_{\mathfrak{g}}(\zeta)_{M}=\left(\xi^{+}-i \eta^{+}-\xi^{-}+i \eta^{-}\right)_{M},
$$

so $\zeta_{M}$ is tangent to $M^{\tau}$ if and only if $\tau_{*}\left(\zeta_{M}\right)=\zeta_{M}$ if and only if $\left(\xi^{-}+i \eta^{+}\right)_{M}=0$, i.e. $\zeta_{M}=\left(\xi^{+}+i \eta^{-}\right)_{M} \in T_{m}(G m)$.

5.6. Example (real flag varieties). Let $M$ be a symmetric coadjoint orbit, $M=$ $U \lambda$ with $\lambda \in \mathfrak{q}^{*}$. We saw in Example 2.9 that $M^{\tau}$ consists of the single $K$-orbit $K \lambda$ and in the proof of Theorem 4.8 that $M$ has a natural compatible complex structure. Therefore $G$ acts in a natural way on $M$ and $M^{\tau}$ consists of a single $G$-orbit. As explained in [6, §5], the stabilizer $G_{\lambda}$ is the real parabolic subgroup $K_{\lambda} \exp i \mathfrak{a} \exp \mathfrak{n}$, where $\mathfrak{n}$ is the nilpotent algebra $\mathfrak{g} \cap \bigoplus_{\alpha \in R_{-} \backslash R_{-}^{\prime}} \mathbb{C} E_{\alpha}$. Thus $M^{\tau}$ is a generalized flag variety of the real reductive group $G$. See further Example 5.14 and Section 9 .

We now explain the relationship between highest-weight sets and polytopes.

5.7. Theorem. Assume that $M^{\tau}$ is nonempty. Then the highest-weight $\operatorname{set} \mathcal{C}(M)$ is equal to the set of rational points in $\Delta(M)$, and $\Delta(M)$ is equal to the closure of $\mathcal{C}(M)$ in $\mathfrak{t}_{+}^{*}$. The same statement holds with $M$ replaced by $M^{\tau}$. Therefore $\Delta\left(M^{\tau}\right)=\Delta(M) \cap \mathfrak{a}^{*}$ and $\Delta\left(M^{\tau}\right)$ is a rational convex polytope.

Proof. The first assertion is proved in 19, 27]; cf. also [5]. The proof of the second assertion follows the same pattern. We begin by proving that $\mathcal{C}\left(M^{\tau}\right)$ is equal to the set of rational points in $\Delta\left(M^{\tau}\right)$. As a special case we show first that

$$
0 \in \mathcal{C}\left(M^{\tau}\right) \quad \Longleftrightarrow \quad 0 \in \Delta\left(M^{\tau}\right) \text {. }
$$


Assume that $0 \in \mathcal{C}\left(M^{\tau}\right)$. Pick $n>0$ and a $U$-invariant $s \in \Gamma\left(M, L^{n}\right)$ such that $s(m) \neq 0$ for some $m \in M^{\tau}$. Choose a $U$-invariant inner product on $\mathfrak{u}$. Then

$$
\operatorname{grad}\|\Phi(m)\|^{2}=2 J \Phi(m)_{M, m}^{b},
$$

where $b: \mathfrak{u}^{*} \rightarrow \mathfrak{u}$ is the linear isomorphism defined by the inner product, and $\Phi(m)_{M}^{b}$ is the vector field on $M$ induced by $\Phi(m)^{b}$. (See e.g. [16, §6].) Let $\mathcal{F}$ be the flow of the vector field $-\operatorname{grad}\|\Phi\|^{2}$ and let $f$ be the function $\langle s, s\rangle$. An easy calculation using (5.4) shows that

$$
\frac{d}{d t} f(\mathcal{F}(t, m))=8 \pi\|\Phi(\mathcal{F}(t, m))\|^{2} f(\mathcal{F}(t, m)) \geq 0 .
$$

Let $m_{\infty}=\lim _{t \rightarrow \infty} \mathcal{F}_{t}(m)$. Then $($ 5.5 $)$ and the fact that $f(m)>0$ imply that $f\left(m_{\infty}\right)>0$. Moreover, $\lim _{t \rightarrow \infty} d f(\mathcal{F}(t, m)) / d t=0$ and hence

$$
\Phi\left(m_{\infty}\right)=0 .
$$

Furthermore, $\Phi(m) \in \mathfrak{q}^{*}$ because $m \in M^{\tau}$, so $i \Phi(m)^{b} \in i \mathfrak{q}=\mathfrak{p}$. Together with (5.4) this implies that $\operatorname{grad}\|\Phi(m)\|^{2}$ is tangent to the orbit $G m$. This orbit is contained in $M^{\tau}$ by Proposition 5.5. The conclusion is that the flow $\mathcal{F}$ preserves the submanifold $M^{\tau}$ and also the $G$-orbits inside it. This means that $m_{\infty} \in \overline{G m} \subseteq M^{\tau}$ and hence, by (5.6) $), 0 \in \Delta\left(M^{\tau}\right)$.

Conversely, if $0 \in \Delta\left(M^{\tau}\right)$ then clearly $0 \in \Delta(M)$, so $0 \in \mathcal{C}(M)$ by the first part of this theorem. Hence $0 \in \mathcal{C}\left(M^{\tau}\right)$ by Lemma 5.3. This concludes the proof of (5.3).

To prove that $\lambda / n \in \mathcal{C}\left(M^{\tau}\right)$ if and only if $\lambda / n \in \Delta\left(M^{\tau}\right)$, where $\lambda \in \Lambda_{+}^{*}$ and $n>0$, we use the shifting trick as in the proof of Lemma 5.3. We note that $\lambda / n \in \Delta\left(M^{\tau}\right)$ if and only if $0 \in \Delta\left(M^{\tau} \times K \lambda^{*}\right)$, and that $\lambda / n \in \mathcal{C}\left(M^{\tau}\right)$ if and only if $0 \in \mathcal{C}\left(M^{\tau} \times K \lambda^{*}\right)$. (Here we have multiplied the symplectic form on $M$ by $n$ and raised its prequantum line bundle to the $n$-th power). Now apply the previous argument to the product $M^{\tau} \times K \lambda^{*}$.

Next we show that $\mathcal{C}\left(M^{\tau}\right)$ is dense in $\Delta\left(M^{\tau}\right)$. This follows from

$$
\overline{\mathcal{C}\left(M^{\tau}\right)}=\overline{\mathcal{C}(M)} \cap \mathfrak{a}^{*}=\Delta(M) \cap \mathfrak{a}^{*} \supseteq \Delta\left(M^{\tau}\right),
$$

where in the first equality we have used that the subspace $\mathfrak{a}^{*}$ of $\mathfrak{t}^{*}$ is defined over the rationals and in the second equality that the closure of $\mathcal{C}(M)$ is $\Delta(M)$. This proves the second statement of the theorem.

The last statement follows immediately from the first two and Lemma 5.3 .

5.8. Remark. Let $\sigma_{+}$be the involution of $\mathbf{t}^{*}$ defined by $\sigma_{+}=-w_{0}^{\prime} \circ \sigma$. It is easy to see that $\sigma_{+}$maps $\Delta(M)$ into itself, and it follows from Lemma 4.7 (囯) that $\Delta\left(M^{\tau}\right)$ is contained in $\Delta(M)^{\sigma_{+}}$. However, if $w_{0}^{\prime} \neq 1$, then $\Delta(M)^{\sigma_{+}}$may be strictly larger than $\Delta\left(M^{\tau}\right)$.

Theorem 5.7 can be generalized to certain singular subsets of $M^{\tau}$.

5.9. Definition. A $(U, \sigma)$-pair in $M$ is a pair $(X, Y)$ of subsets $X \subseteq M$ and $Y \subseteq M^{\tau}$ subject to the following requirements: $X$ is a $U$-stable and $\tau$-stable irreducible closed complex algebraic subvariety of $M$ such that $X_{\text {reg }}$ intersects $M^{\tau}$, and $Y$ is the closure of $S$, where $S$ is any connected component of $X_{\operatorname{reg}} \cap M^{\tau}$. 
Here $X_{\text {reg }}$ denotes the set of smooth points of $X$. Standard results in real algebraic geometry (see e.g. [2, Ch. 2]) say that $X_{\text {reg }} \cap M^{\tau}$ has only finitely many connected components and that $Y$ is a semi-algebraic subset of $M^{\tau}$. It follows from Proposition 5.5 and the fact that $X_{\text {reg }}$ is $U^{\mathbb{C}}$-stable that $S$, and hence $Y$, are stable under the action of $G$. It is obvious how to extend the definitions of $\Delta$ and $\mathcal{C}$ to this setting: one puts $\Delta(X)=\Phi(X) \cap \mathfrak{t}_{+}^{*}$ and $\Delta(Y)=\Phi(Y) \cap \mathfrak{t}_{+}^{*}$; and in the definition of $\mathcal{C}(M)$ and $\mathcal{C}\left(M^{\tau}\right)$ one replaces $M$ by $X, M^{\tau}$ by $Y$, and $L$ by $\left.L\right|_{X}$.

5.10. Theorem. Let $(X, Y)$ be any $(U, \sigma)$-pair in $M$. Then Theorem 5.7 remains valid if we replace $M$ by $X$ and $M^{\tau}$ by $Y$.

Proof. This is proved in exactly the same way as Theorem 5.7. The hypotheses on $X$ and $Y$ imply that $Y$ contains a Lagrangian submanifold of $X_{\text {reg. }}$. Therefore $Y$ is Zariski dense in $X$, that is to say, the smallest complex subvariety of $M$ containing $Y$ is equal to $X$. This ensures that a section of $\left.L\right|_{X}$ which vanishes on $Y$ vanishes on $X$. The fact that $Y$ is $G$-stable implies that the flow $\mathcal{F}$ maps $Y$ into itself. Since $Y$ is closed the limit of every trajectory in $Y$ is contained in $Y$. These facts guarantee that the arguments used to prove Lemma 5.3 and Theorem 5.7 still work in the present situation.

For instance, let $X=M$ and let $Y$ be any connected component of $M^{\tau}$. Then $(X, Y)$ is a $(U, \sigma)$-pair.

5.11. Corollary. If $Y$ is a connected component of $M^{\tau}$, then $\Delta(Y)=\Delta(M) \cap \mathfrak{a}^{*}$.

More interesting examples are obtained by considering any point $m$ in $M^{\tau}$ and letting $X$ be the closure of the orbit $U^{\mathbb{C}} m$. Then $X_{\text {reg contains }} U^{\mathbb{C}} m$, so we can take $Y$ to be the closure of any component of $U^{\mathbb{C}} m \cap M^{\tau}$, for instance $Y=\overline{G m}$. The conclusion is as follows.

5.12. Corollary (orbit closures). $\Delta(\overline{G m})=\Delta\left(\overline{U^{\mathbb{C}} m}\right) \cap \mathfrak{a}^{*}$ for every $m \in M^{\tau}$.

This is a generalization to the nonabelian case of Theorem 2 in Atiyah's paper [1. Brion [5] proved that for "most" $m$ the moment polytope of the orbit closure $\frac{U^{\mathbb{C}} m}{}$ is equal to the full moment polytope $\Delta(M)$. We show now that a similar result holds for the real orbits $G m$. This was first pointed out by Duistermaat [6] in the abelian case. Our proof is patterned on Brion's. If $\mathbb{K}$ denotes the field $\mathbb{R}$ or $\mathbb{C}$, let us refer to open subsets in the Zariski topology over $\mathbb{K}$ as $\mathbb{K}$-Zariski open.

5.13. Corollary. For all $(U, \sigma)$-pairs $(X, Y)$ there exists a nonempty $\mathbb{C}$-Zariski open subset $O$ of $X$ such that $\Delta(Y)=\Delta(\overline{G m})$ for all $m \in O \cap Y$. The set $O \cap Y$ is dense in $Y$.

The second statement does not follow automatically from the first, because $\mathbb{R}$ Zariski open subsets of an irreducible real algebraic variety are not necessarily dense. They are always dense, however, if the variety is nonsingular.

Proof. Let $\xi_{1}, \xi_{2}, \ldots, \xi_{k} \in \mathfrak{a}^{*}$ be the vertices of the polytope $\Delta(Y)$. For each $i$ select $\lambda_{i} \in \Lambda_{+}^{*} \cap \mathfrak{a}^{*}$ and $n_{i}>0$ such that $\xi_{i}=\lambda_{i} / n_{i}$ and pick a nonzero $U$-invariant $s_{i} \in \Gamma\left(X, L^{n}\right)$. Let $O$ be the $\mathbb{C}$-Zariski open subset of $X$ consisting of all $m \in X$ such that $s_{i}(m) \neq 0$ for all $i$. For every $m \in O \cap Y, s_{i}(m) \neq 0$ and therefore $\xi_{i} \in \mathcal{C}(\overline{G m})$ for all $i$. Using Theorem 5.10 we conclude that $\Delta(Y) \subseteq \Delta(\overline{G m})$. On the other hand, it is plain that $\Delta(\overline{G m}) \subseteq \Delta(Y)$ and so the two polytopes are equal. 
The subset $O$ intersects the real algebraic variety $X_{\text {reg }} \cap M^{\tau}$ in an $\mathbb{R}$-Zariski open subset and it intersects each component $S$ of $X_{\text {reg }} \cap M^{\tau}$ nontrivially since $S$ is a totally real Lagrangian in $X_{\text {reg }}$. Added to the fact that $X_{\text {reg }} \cap M^{\tau}$ is nonsingular, this implies that $O \cap X_{\text {reg }} \cap M^{\tau}$ is dense in $X_{\text {reg }} \cap M^{\tau}$. Consequently $O \cap Y$ is dense in $Y$.

5.14. Example (products of orbits). Let $M=U \lambda \times U \mu$ be the product of the coadjoint orbits through $\lambda, \mu \in \mathfrak{a}_{+}^{*}$. Then $M^{\tau}=K \lambda \times K \mu$. Let us assume for simplicity that $\lambda$ and $\mu$ are generic, so that $U_{\lambda}=U_{\mu}=\mathcal{Z}_{K}(A)=K^{\prime}$. According to Example 5.6 we can identify $M^{\tau}$ with $G / B \times G / B$, where $B$ is the real Borel subgroup $K^{\prime} \exp i \mathfrak{a} \exp \mathfrak{n}$ of $G$. We see immediately that the $G$-orbits on $G / B \times G / B$ are in a natural one-to-one correspondence with the $B$-orbits on $G / B$. Moreover, this correspondence preserves the closure relation among orbits. Applying the Bruhat decomposition (Theorem 1.4 in [12, Ch. IX]) we obtain the following result: the $G$-orbits $\mathcal{O}_{w}$ on $M^{\tau}$ are of the form

$$
\mathcal{O}_{w}=G(\overline{1}, \bar{w})
$$

for $w \in W^{\mathfrak{a}}$; and $\mathcal{O}_{v}$ is in the closure of $\mathcal{O}_{w}$ if and only if $v \leq w$. Here $\bar{w}$ denotes the coset $n \bmod B \in G / B$ determined by any representative $n \in \mathcal{N}_{K}(A)$ of $w \in W^{\mathfrak{a}}$, and " $\leq$ " denotes the Bruhat-Chevalley ordering on $W^{\mathfrak{a}}$. Thus there is exactly

one closed orbit, the diagonal $\mathcal{O}_{1}$, and exactly one open orbit, $\mathcal{O}_{w_{0}^{a}}$. It is clear that $\Delta\left(\mathcal{O}_{1}\right)=\{\lambda+\mu\}$ and $\Delta\left(\overline{\mathcal{O}}_{w_{0}^{\mathfrak{a}}}\right)=\Delta\left(M^{\tau}\right)$. See Section 9 for a complete calculation of $\Delta\left(M^{\tau}\right)$. Let us here calculate it and its subpolytopes $\Delta\left(\overline{\mathcal{O}}_{w}\right)$ under the simplifying assumption that $\Delta\left(M^{\tau}\right)$ is entirely contained in the interior of $\mathfrak{t}_{+}^{*}$. Then Theorem 3.1 implies that all vertices of $\Delta\left(M^{\tau}\right)$ are images under $\Phi$ of $A$-fixed points on $K \lambda \times K \mu$. According to Lemma B.4(ii) the set of $A$-fixed points is equal to $W^{\mathfrak{a}} \lambda \times W^{\mathfrak{a}} \mu$, and therefore

$$
\Delta\left(M^{\tau}\right)=\operatorname{hull}\left\{u \lambda+v \mu \mid u, v \in W^{\mathfrak{a}}, u \lambda+v \mu \text { dominant }\right\} .
$$

Likewise, the vertices of $\Delta\left(\overline{\mathcal{O}}_{w}\right)$ are images of $A$-fixed points on $\overline{\mathcal{O}}_{w}=\bigcup_{v \leq w} \mathcal{O}_{v}$. An easy computation yields that the $A$-fixed points on $\mathcal{O}_{v}$ are the points $n(\overline{1}, \bar{v})$ with $n \in \mathcal{N}_{K}(A)$. Thus

$$
\Delta\left(\overline{\mathcal{O}}_{w}\right)=\operatorname{hull}\left\{u(\lambda+v \mu) \mid u, v \in W^{\mathfrak{a}}, v \leq w, u(\lambda+v \mu) \text { dominant }\right\} .
$$

\section{THE AFFINE CASE}

Before we treat the general case we prove a version of the Lagrangian convexity theorem for affine varieties. This is not really a special case of Theorem 3.1, because the moment map on an affine variety is not proper, but it is needed in the proof of Theorem 3.1. Also, as in the projective case, the result holds for singular as well as nonsingular varieties, for instance closures of orbits of the noncompact real reductive group $G$. The idea of the proof is the same as in the projective case.

Let $(V, \tau)$ be a (finite-dimensional) unitary $(U, \sigma)$-module and let $M$ be a $U$ stable and $\tau$-stable irreducible affine subvariety of $V$.

6.1. Definition. The highest-weight set of $M$ is the set $\mathcal{C}(M)$ consisting of all dominant weights $\lambda$ such that the irreducible representation $V_{\lambda}$ occurs in the coordinate ring $\mathbb{C}[M]$. The highest-weight set of $M^{\tau}$ is the $\operatorname{set} \mathcal{C}\left(M^{\tau}\right)$ of all $\lambda \in \Lambda_{+}^{*} \cap \mathfrak{a}^{*}$ such that there exist $m \in M, k \in K$, and a $U$-invariant global algebraic section $s$ of $L \otimes L_{\lambda^{*}}$ with $s\left(m, k \lambda^{*}\right) \neq 0$. 
Here $L$ denotes the trivial line bundle on $M$ with the trivial lift of the $U$-action. The proof of the following result is similar to that of Lemma 5.3.

6.2. Lemma. If $M^{\tau}$ contains a smooth point of $M$, then $\mathcal{C}\left(M^{\tau}\right)=\mathcal{C}(M) \cap \mathfrak{a}^{*}$.

Proposition 5.5 is valid exactly as stated in the present situation. Here is the analogue of Theorem 5.7.

6.3. Theorem. Assume that $M^{\tau}$ contains a smooth point of $M$. Then hull $\mathbb{Q} \mathcal{C}(M)$ is equal to the set of rational points in $\Delta(M)$, and $\Delta(M)$ is equal to the closure of hull $\mathbb{Q}_{\mathbb{C}} \mathcal{C}(M)$ in $\mathfrak{t}_{+}^{*}$. The same statement holds with $M$ replaced by $M^{\tau}$. Therefore $\Delta\left(M^{\tau}\right)=\Delta(M) \cap \mathfrak{a}^{*}$ and $\Delta\left(M^{\tau}\right)$ is a rational convex polyhedral cone.

Here "hull $\mathbb{Q}$ " denotes the convex hull of a subset of a vector space over $\mathbb{Q}$. The assumption that $M$ is irreducible implies that $\mathcal{C}(M)$ is closed under addition, and therefore hull $\mathbb{Q} \mathcal{C}(M)$, resp. hull $\mathbb{Q} \mathcal{C}\left(M^{\tau}\right)$, is the set of all positive rational multiples of elements of $\mathcal{C}(M)$, resp. $\mathcal{C}\left(M^{\tau}\right)$.

Proof. See [28, Theorem 4.9] for the first statement. Next, let us show that hull $_{\mathbb{Q}} \mathcal{C}\left(M^{\tau}\right)$ is a subset of $\Delta\left(M^{\tau}\right)$. Let $\xi \in \operatorname{hull}_{\mathbb{Q}} \mathcal{C}\left(M^{\tau}\right)$; then $\lambda=n \xi \in \mathcal{C}\left(M^{\tau}\right)$ for some positive integer $n$. Consider the product $M^{\prime}=M \times U \lambda^{*}$ equipped with the symplectic form $\omega^{\prime}=n \omega+\omega_{\lambda^{*}}$ and the moment map $\Phi^{\prime}=n \Phi+\iota_{\lambda^{*}}$. Here $\omega_{\lambda}$ denotes the symplectic form on $U \lambda$ and $\iota_{\lambda}$ the inclusion of $U \lambda$ into $\mathfrak{u}^{*}$. We need to show that $0 \in \Delta\left(\left(M^{\prime}\right)^{\tau}\right)$. This is established by picking a $U$-invariant global algebraic section $s$ of $L^{\prime}=L \otimes L_{\lambda^{*}}$ and a point $m^{\prime}=(m, k \lambda) \in\left(M^{\prime}\right)^{\tau}$ with $s\left(m^{\prime}\right) \neq 0$, and considering the trajectory of $m^{\prime}$ under the flow $\mathcal{F}^{\prime}$ of $-\operatorname{grad}\left\|\Phi^{\prime}\right\|^{2}$. It is proved in 28] that $m_{\infty}^{\prime}=\lim _{t \rightarrow \infty} \mathcal{F}_{t}^{\prime}\left(m^{\prime}\right)$ exists. One then shows as in the proof of Theorem 5.7 that $m_{\infty}^{\prime} \in\left(M^{\prime}\right)^{\tau}$ and $\Phi^{\prime}\left(m_{\infty}^{\prime}\right)=0$. (One uses the Hermitian metric on $L$ defined by the Gaussian $\exp \left(-\pi n\|\cdot\|^{2}\right)$.)

Now assume that $\xi$ is a rational point in $\Delta\left(M^{\tau}\right)$. Then $\xi \in \Delta(M) \cap \mathfrak{a}^{*}$, so, by the first part of the theorem $\xi \in \operatorname{hull}_{\mathbb{Q}} \mathcal{C}(M) \cap \mathfrak{a}^{*}$, which is equal to hull $\mathbb{Q} \mathcal{C}\left(M^{\tau}\right)$ by Lemma 6.2. We have shown that $\Delta(M) \cap\left(\Lambda^{*} \otimes \mathbb{Q}\right)=\operatorname{hull}_{\mathbb{Q}} \mathcal{C}\left(M^{\tau}\right)$.

The fact that hull $\mathbb{Q} \mathcal{C}\left(M^{\tau}\right)$ is dense in $\Delta\left(M^{\tau}\right)$ is proved as in (5.7).

The last assertion of the theorem follows from the first two plus Lemma 6.2.

6.4. Corollary. The moment cones $\Delta(M)$ and $\Delta\left(M^{\tau}\right)$ are independent of the $U \rtimes\{ \pm 1\}$-equivariant affine embedding of $M$ into the $(U, \sigma)$-module $V$.

We leave it to the reader to state and prove analogues of Corollaries 5.12 and 5.13 , and finish this section with a necessary criterion for the origin to be an extreme point of the moment cone.

6.5. Lemma. Assume that $\Delta\left(M^{\tau}\right)$ is a proper cone. Then $A \subseteq[U, U] U_{m}$ for all $m$ such that $U^{\mathbb{C}} m$ is closed in $M$.

Proof. Consider the homogeneous variety $X=U^{\mathbb{C}} /\left[U^{\mathbb{C}}, U^{\mathbb{C}}\right] U_{m}^{\mathbb{C}}$. The closedness of the orbit $U^{\mathbb{C}} m$ ensures that $\left(U^{\mathbb{C}}\right)_{m}$ is the complexification of $U_{m}$ and that $X$ is affine. According to the proof of 28 , Theorem 4.25], $\Delta(X)$ is contained in $\Delta(M)$ and is equal to the kernel of the projection $p: \mathfrak{u}^{*} \rightarrow\left([\mathfrak{u}, \mathfrak{u}]+\mathfrak{u}_{m}\right)^{*}$. Therefore, if $\Delta\left(M^{\tau}\right)=\Delta(M) \cap \mathfrak{a}^{*}$ is a proper cone, then ker $p \cap \mathfrak{a}^{*}=\{0\}$. Hence $p$ induces an injection $\mathfrak{a}^{*} \hookrightarrow\left([\mathfrak{u}, \mathfrak{u}]+\mathfrak{u}_{m}\right)^{*}$, and so $\mathfrak{a} \subseteq[\mathfrak{u}, \mathfrak{u}]+\mathfrak{u}_{m}$. This is equivalent to $A \subseteq[U, U] U_{m}$ 


\section{NORMAL FORMS}

In this section we extend to the category of Hamiltonian $(U, \sigma)$-manifolds a wellknown local normal form theorem of Guillemin and Sternberg [11] and Marle [23]. Because this is mostly a routine exercise, which amounts to inserting $\sigma \mathrm{s}$ and $\tau \mathrm{s}$ in the right places, we omit the proof. The techniques involved are a straightforward combination of those used in loc. cit., Meyer [26], and Duistermaat [6].

Let $H$ be a closed (but not necessarily connected) $\sigma$-stable subgroup of $U$ and let $V$ be a finite-dimensional unitary $(H, \sigma)$-module, considered as a Hamiltonian $(H, \sigma)$-manifold with momentum map $\Phi_{V}$ as in Example 2.7. According to Examples 2.6 and 2.13 the product $T^{*} U \times V$ is a Hamiltonian $(U \times H, \sigma \times \sigma)$ manifold, where $U$ acts on $T^{*} U$ by left multiplication, $H$ acts on $T^{*} U$ by right multiplication and linearly on $V$. Therefore, by Example 2.10, the symplectic quotient $\left(T^{*} U \times V\right) / / H$ is a Hamiltonian $(U, \sigma)$-manifold. There is a naturally defined $U$-equivariant diffeomorphism between $\left(T^{*} U \times V\right) / / H$ and the associated bundle

$$
F(H, V)=U \times{ }^{H}\left(\mathfrak{h}^{0} \times V\right),
$$

which depends only on the choice of a connection on the principal $H$-bundle $U \rightarrow$ $U / H$. Here $\mathfrak{h}^{0}$ denotes the annihilator of $\mathfrak{h}$ in $\mathfrak{u}^{*}$. (See e.g. [25, Appendix A].)

Now let $M$ be an arbitrary Hamiltonian $(U, \sigma)$-manifold and let $m \in \Phi^{-1}(0)$. Let $H$ be the stabilizer of $m$ and $V=T_{m}(U m)^{\omega} / T_{m}(U m)$ the symplectic slice at $m$. It is expedient to choose a $U$-invariant and $\sigma$-anti-invariant compatible almost complex structure on $M$; then the symplectic slice is a unitary representation in a natural way. Moreover, if $\tau(m)=m$, the subgroup $H$ is $\sigma$-stable, the subspace $\mathfrak{h}^{0}$ of $\mathfrak{u}^{*}$ is $\sigma$-stable, and $V$ is a unitary $(H, \sigma)$-module. We can now state the local normal form theorem.

7.1. Theorem (symplectic slices). Let $m \in \Phi^{-1}(0) \cap M^{\tau}$ and $H=U_{m}$. Let $V$ be the symplectic slice at $m$. Then there exists a $U \rtimes\{ \pm 1\}$-stable open neighbourhood of $m$ in $M$ which is isomorphic as a Hamiltonian $(U, \sigma)$-manifold to a neighbourhood of the zero section in $F(H, V)$. The isomorphism maps $m$ to $[1,0,0]$; the moment map and the involution on $F(H, V)$ are given by

$$
\begin{aligned}
& \Phi:[u, \xi, v] \longmapsto u\left(\xi+\Phi_{V}(v)\right), \\
& \tau:[u, \xi, v] \longmapsto\left[\sigma(u),-\sigma(\xi), \tau_{V}(v)\right],
\end{aligned}
$$

respectively, where $\tau_{V}$ denotes the involution induced on $V$.

One interesting consequence of this result concerns the symplectic quotient $M / / U$. The involution $\tau$ preserves the fibre $\Phi^{-1}(0)$ and maps $U$-orbits to $U$-orbits. It therefore descends to an involution on $M / / U$, which we denote also by $\tau$. If $U$ acts freely on $\Phi^{-1}(0)$, then $M / / U$ is a symplectic manifold and the involution is antisymplectic. Therefore the fixed-point set $(M / / U)^{\tau}$ is a Lagrangian submanifold. The Lagrangian quotient or Lagrangian reduced space $M^{\tau} / / U$ is by definition the space obtained by projecting $U M^{\tau} \cap \Phi^{-1}(0)$ into $M / / U$.

7.2. Corollary. If $U$ acts freely on $\Phi^{-1}(0)$, then the Lagrangian quotient $M^{\tau} / / U$ is a union of connected components of the Lagrangian $(M / / U)^{\tau}$.

Proof. Clearly both $M^{\tau} / / U$ and $(M / / U)^{\tau}$ are closed and $M^{\tau} / / U \subseteq(M / / U)^{\tau}$, so it suffices to show that $M^{\tau} / / U$ is a Lagrangian submanifold. Let $m \in M^{\tau}$. We 
compute in the model $F(H, V)$. Writing $\mathfrak{h}^{0}=\mathfrak{m}$ we have an inclusion

$$
U^{\sigma} \times H^{\sigma}\left(\mathfrak{m}^{-\sigma} \times V^{\tau}\right) \subseteq F(H, V)^{\tau}
$$

of closed Lagrangian submanifolds of $F(H, V)$. Upon taking quotients we obtain

$$
\left(V^{\tau} \cap \Phi_{V}^{-1}(0)\right) / H^{\sigma} \subseteq F(H, V)^{\tau} / / U .
$$

Now we use the assumption that $U$ acts freely. This implies that $H=\{1\}$, $\Phi_{V}=0$, and also that the inclusions (7.1) and (7.2) are equalities. Therefore $F(H, V) / / U=V$ and $F(H, V)^{\tau} / / U=V^{\tau}$. The upshot is that $F(H, V)^{\tau} / / U$ is a Lagrangian submanifold of $F(H, V) / / U$.

7.3. Remark (singular quotients). If we drop the assumption that $U$ acts freely on $\Phi^{-1}(0)$, the symplectic quotient $M / / U$ is a symplectic stratified space. An argument analogous to the proof of Corollary 7.2 shows that the decomposition of $M / / U$ into symplectic strata induces a decomposition of the Lagrangian quotient $M^{\tau} / / U$ into smooth manifolds, each of which is Lagrangian in the ambient symplectic stratum of $M / / U$. Moreover, $M^{\tau} / / U$ always intersects the open stratum of $M / / U$, so that $\operatorname{dim} M^{\tau} / / U=\frac{1}{2} \operatorname{dim} M / / U$.

Another consequence of the normal form theorem is the fact that $M$ is locally, near the orbit through any point $m \in M^{\tau} \cap \Phi^{-1}(0)$, isomorphic to an affine algebraic Hamiltonian $(U, \sigma)$-manifold. As before, let $H=U_{m}$ and let $V$ be the symplectic slice at $m$. Consider the associated bundle $U^{\mathbb{C}} \times{ }^{\mathbb{C}} V$, which is an affine variety with a natural algebraic action of $U^{\mathbb{C}}$.

7.4. Lemma. There exist a finite-dimensional unitary $(U, \sigma)$-module $(E, \tau)$ and a $U$-equivariant algebraic embedding $\iota: U^{\mathbb{C}} \times{ }^{\mathbb{C}} V \hookrightarrow E$ such that the image $X=$ $\iota\left(U^{\mathbb{C}} \times{ }^{\mathbb{C}} V\right)$ is $\tau$-stable. Furthermore, if $x=\iota([1,0])$, then $\Phi_{E}(x)=0$ and the symplectic slice at $x$ (relative to the symplectic structure on $X$ induced by the constant symplectic form on $E$ ) is isomorphic to $V$.

Outline of proof. Without the $\tau$, this is [28, Example 5.5]. Let us show briefly how to adapt the argument to the present context.

We use the fact that for every compact Lie group $\mathcal{G}$ and closed subgroup $\mathcal{H}$ there exists a finite-dimensional real $\mathcal{G}$-module with a vector in it whose stabilizer is equal to $\mathcal{H}$. Taking $\mathcal{G}=U \rtimes\{ \pm 1\}$ and $\mathcal{H}=H \rtimes\{ \pm 1\}$, where $\{ \pm 1\}$ acts on $U$ and $H$ by means of the involution $\sigma$, gives us a real $U$-module $E_{1}^{\mathbb{R}}$ that is equipped with an involution $\tau_{1}^{\mathbb{R}}$ and contains a vector $e_{1}$ such that $U_{e_{1}}=H, \tau_{1}^{\mathbb{R}}\left(e_{1}\right)=e_{1}$, and $\tau_{1}^{\mathbb{R}}(u e)=\sigma(u) e$ for all $u \in U$ and $e \in E_{1}^{\mathbb{R}}$. Let $E_{1}$ be the complexification of $E_{1}^{\mathbb{R}}$ and $\tau_{1}$ the conjugate linear extension of $\tau_{1}^{\mathbb{R}}$ to $E_{1}$, i.e. the complex linear extension composed with complex conjugation. Then $\left(E_{1}, \tau_{1}\right)$ is a unitary $(U, \sigma)$-module, and the map $U^{\mathbb{C}} \rightarrow E_{1}$ which sends $g$ to $g e_{1}$ descends to a closed equivariant algebraic embedding $\iota_{1}: U^{\mathbb{C}} / H^{\mathbb{C}} \hookrightarrow E_{1}$. The image of $\iota_{1}$ is $\tau_{1}$-stable: if $g \in U^{\mathbb{C}}$, then $g=u \exp (i \xi)$ with $u \in U$ and $\xi \in \mathfrak{u}$, so

$$
\tau_{1}\left(g e_{1}\right)=\tau_{1}\left(u \exp (i \xi) e_{1}\right)=\sigma(u \exp (-i \xi)) \tau_{1}\left(e_{1}\right) \in U^{\mathbb{C}} e_{1} .
$$

Moreover, there exists a (complex, finite-dimensional) $(U, \sigma)$-module $\left(E_{2}, \tau_{2}\right)$ together with an embedding $\iota_{2}: V \hookrightarrow E_{2}$ which intertwines the $H$-actions and the involutions. To prove this, by virtue of the structure theorem, Addendum 4.11, we may assume $V$ to be either of type 4.8 ) or (4.9). (See Remark 4.12 if $H$ is not connected.) Let $F$ be a (complex, finite-dimensional) $(U, \sigma)$-module containing a 
vector whose stabilizer is $U$. Let $\lambda$ be a dominant weight of $U$. An argument based on Peter-Weyl (see e.g. [14) shows that there exists an $H$-equivariant embedding $j: V_{\lambda} \hookrightarrow E_{2}$, where $E_{2}$ is of the form $F \otimes \cdots \otimes F \otimes F^{*} \otimes \cdots \otimes F^{*}$. Observe that $E_{2}$ is a $(U, \sigma)$-module with involution $\tau_{2}$ in a natural way. Assume that $V=V_{\lambda}$ is of type (4.8). Put $\iota_{2}=\frac{1}{2}\left(j+\tau_{2} \circ j \circ \tau\right)$. Then $\iota_{2}$ is $H$-equivariant and intertwines the involutions. By Schur's Lemma, $\iota_{2}$ is either injective or 0. In the first case we are done; in the second case we replace $\tau_{2}$ by $-\tau_{2}$ and $\iota_{2}$ by $\frac{1}{2}\left(j-\tau_{2} \circ j \circ \tau\right)$. Now assume that $V=V_{\lambda}^{\text {dup }}$ is of type (4.9). Then the intersection of $j\left(V_{\lambda}\right)$ and $\tau\left(j\left(V_{\lambda}\right)\right)$ is $\{0\}$, so $\iota_{2}=j \oplus \tau_{2} \circ j \circ \tau$ defines an $H$-equivariant embedding of $V \cong V_{\lambda} \oplus V_{\sigma_{+}(\lambda)}$ into $E_{2}$ which intertwines $\tau$ and $\tau_{2}$.

Finally, we embed the associated bundle into $E_{1} \oplus E_{2}$ by putting $\iota([g, v])=$ $\left(g e_{1}, g \iota_{2}(v)\right)$.

The upshot of Theorem 7.1 and Lemma 7.4 is as follows.

7.5. Corollary. Under the hypotheses of Theorem 7.1 there exists a $U \rtimes\{ \pm 1\}$ stable open neighbourhood of $m$ in $M$ which is isomorphic as a Hamiltonian $(U, \sigma)$ manifold to a neighbourhood of $x$ in $X$.

\section{Proof of the main theorem}

Let $M$ be an arbitrary $(U, \sigma)$-manifold and let $m \in M^{\tau}$. Assume that $\lambda=\Phi(m)$ is in the fundamental Weyl chamber. The positive roots of $\left(U_{\lambda}, T\right)$ are by definition those positive roots of $(U, T)$ which are perpendicular to $\lambda$, and the fundamental Weyl chamber $\mathfrak{t}_{+, \lambda}^{*}$ of $U_{\lambda}$ is chosen accordingly. Let $H=U_{m} \subseteq U_{\lambda}$ be the stabilizer of $m$ and

$$
V=T_{m}(U m)^{\omega} /\left(T_{m}(U m) \cap T_{m}(U m)^{\omega}\right)
$$

the symplectic slice at $m$. Consider the affine $\left(U_{\lambda}, \sigma\right)$-variety $X=U_{\lambda}^{\mathbb{C}} \times{ }^{\mathbb{C}} V$ and its moment cone $\Delta(X) \subseteq \mathfrak{t}_{+, \lambda}^{*}$.

8.1. Definition. The local moment cone of $M$ at $m$ is $\Delta_{m}=\lambda+\Delta(X)$. The local moment cone of $M^{\tau}$ at $m$ is $\Delta_{m}^{\tau}=\lambda+\Delta\left(X^{\tau}\right)$.

It follows from Theorem 6.3 that $\Delta_{m}^{\tau}=\Delta_{m} \cap \mathfrak{a}^{*}$. Here is a local version of Theorem 3.1 .

8.2. Theorem. For every sufficiently small $U$-stable and $\tau$-stable open neighbourhood $O$ of $m$ the set $\Delta(O)$ is a neighbourhood of the vertex $\lambda$ in $\Delta_{m}$, and $\Delta\left(O^{\tau}\right)$ is a neighbourhood of the vertex $\lambda$ in $\Delta_{m}^{\tau}$.

Proof. The first statement is [28, Theorem 6.5]. We now prove the second statement. Under the assumption that $\lambda=0$ this follows from Corollary 7.5, together with the following observation: if $O$ is a $U$-stable and $\tau$-stable neighbourhood of $x$ in $X$, then $\Delta\left(O^{\tau}\right)$ is a neighbourhood of the vertex in $\Delta\left(X^{\tau}\right)$. This would be automatic if the moment map on $X$ were proper, which it usually is not. However, we can factor $\Phi$ into a proper moment map and a linear projection. The $S^{1}$-action on $X$ defined by complex scalar multiplication on the slice $V$ commutes with the $U$-action and is Hamiltonian with moment map $\phi([g, v])=-\frac{1}{2}\|v\|^{2}$. It is not hard to show that the joint moment map $\Phi \times \phi: X \rightarrow \mathfrak{u}^{*} \times \mathbb{R}$ is proper. Clearly $\Phi=\pi \circ(\Phi \times \phi)$, where $\pi: \mathfrak{u}^{*} \times \mathbb{R} \rightarrow \mathfrak{u}^{*}$ is the projection onto the first factor. For $x \in X$ define $\Phi_{+}(x)$ to be the unique intersection point of the coadjoint orbit $U \Phi(x)$ with the Weyl chamber $\mathfrak{t}_{+}^{*}$. Being proper, $\Phi_{+} \times \phi$ maps $O^{\tau}$ onto a 
neighbourhood of the vertex of the $U \times S^{1}$-moment cone of $X^{\tau}$ and, being linear, $\pi$ projects this neighbourhood onto a neighbourhood of the vertex in $\Delta\left(O^{\tau}\right)$.

The general case can be reduced to the case $\lambda=0$ by means of symplectic crosssections. Let $\mathfrak{s}$ be the (relatively open) face of $\mathfrak{t}_{+}^{*}$ containing $\lambda$. Let $\mathfrak{S}_{\mathfrak{s}}=U_{\mathfrak{s}} \cdot \operatorname{star} \mathfrak{s}$, where star $\mathfrak{s}$ stands for the open star $\bigcup_{\mathfrak{r} \succeq \mathfrak{s}} \mathfrak{r}$ of $\mathfrak{s}$ and $U_{\mathfrak{s}}$ is the centralizer of $\mathfrak{s}$. Then $\mathfrak{S}_{\mathfrak{s}}$ is a maximal slice for the coadjoint action at $\lambda$. Its preimage $M_{\mathfrak{s}}=\Phi^{-1}\left(\mathfrak{S}_{\mathfrak{s}}\right)$ is the symplectic cross-section at $\mathfrak{s}$. (See e.g. [25, Section 3.2].) The fact that $\lambda \in \mathfrak{s} \cap \mathfrak{a}^{*}$ implies that $\mathfrak{s}$, and hence star $\mathfrak{s}$, are stable under $\sigma_{+}$. Moreover, the longest element $w_{0}^{\prime} \in W^{\prime}$ has a representative $k_{0}$ which is in $K^{\prime} \subseteq U_{\mathfrak{s}}$. It follows from this that $-\sigma(u \xi)=\sigma(u) k_{0} \sigma_{+}(\xi) \in \mathfrak{S}_{\mathfrak{s}}$ for all $u \in U_{\mathfrak{s}}$ and $\xi \in$ star $\mathfrak{s}$, i.e. $\mathfrak{S}_{\mathfrak{s}}$ is stable under $-\sigma$. Consequently $M_{\mathfrak{s}}$ is $\tau$-stable and is therefore a Hamiltonian $\left(U_{\mathfrak{s}}, \sigma\right)$-manifold in its own right. Moreover, $\Delta\left(M_{\mathfrak{s}}\right)=\Delta(M) \cap \operatorname{star} \mathfrak{s}$ and $\Delta\left(M_{\mathfrak{s}}^{\tau}\right)=\Delta\left(M^{\tau}\right) \cap \operatorname{star} \mathfrak{s}$ by equivariance of the moment map. It therefore suffices to prove the result for $M_{\mathfrak{s}}$ instead of $M$. Since $\lambda$ is annihilated by $U_{\mathfrak{s}}$, we can subtract $\lambda$ from the moment map to get $\Phi(m)=0$.

Proof of Theorem 3.1. It is clear that $\Delta\left(M^{\tau}\right)$ is contained in $\Delta(M) \cap \mathfrak{a}^{*}$. Let $\lambda \in$ $\Delta\left(M^{\tau}\right)$. According to [28, Theorem 6.5] the properness of $\Phi$ implies that the local moment cone $\Delta_{m}$ is the same for all $m \in \Phi^{-1}(\lambda)$. Hence, by Theorem 6.3, the cone $\Delta_{m}^{\tau}$ is the same for all $m \in \Phi^{-1}(\lambda) \cap M^{\tau}$. Together with Theorem 8.2 this implies that a small neighbourhood of $\lambda$ in $\Delta\left(M^{\tau}\right)$ is equal to a small neighbourhood of $\lambda$ in $\Delta_{m}^{\tau}$. In particular, $\Delta\left(M^{\tau}\right)$ is locally convex. It is also closed because $\Phi$ is proper, and hence it is globally convex. A convex set is the intersection of all convex cones containing it, so

$$
\Delta\left(M^{\tau}\right)=\bigcap_{m \in M^{\tau}} \Delta_{m}^{\tau}=\bigcap_{m \in M^{\tau}} \Delta_{m} \cap \mathfrak{a}^{*} \subseteq \bigcap_{m \in M} \Delta_{m} \cap \mathfrak{a}^{*}=\Delta(M) \cap \mathfrak{a}^{*},
$$

and hence $\Delta\left(M^{\tau}\right)=\Delta(M) \cap \mathfrak{a}^{*}$. This proves (闻).

(iii) follows from Lemma 6.5 and Theorem 8.2 .

\section{Applications}

Cotangent bundles. Let $S$ be a connected manifold equipped with a $U$-action and an involution $\tau$ such that $\tau(u s)=\sigma(u) \tau(s)$. Let $M=T^{*} S$ be the $(U, \sigma)$ manifold defined in Example 2.6. The moment map on $M$ is not proper unless $S$ is a homogeneous $U$-manifold. Nevertheless, Theorem 8.2 enables us to describe the images of $M$ and $M^{\tau}=T_{S^{\tau}}^{*} S$. Assume that $S^{\tau}$ is nonempty and take any point $s$ in it. Let $H$ be the stabilizer $U_{s}$ and $V^{\mathbb{R}}=T_{s} S / T_{s}(U s)$ the slice at $s$. Then $\tau$ induces an involution $\tau^{\mathbb{R}}$ on $V^{\mathbb{R}}$ and the symplectic slice $V$ at $s$ is isomorphic to the complexification of $V^{\mathbb{R}}$, equipped with the obvious $U$-action and the conjugate linear extension of $\tau^{\mathbb{R}}$. The affine local model $U^{\mathbb{C}} \times{ }^{H^{\mathbb{C}}} V$ is isomorphic to the complexification of the real affine $U$-variety $U \times{ }^{H} V^{\mathbb{R}}$.

9.1. Theorem. The set $\Delta\left(T^{*} S\right)$ is a rational convex polyhedral cone. It is equal to the moment cone of the affine $U$-variety $U^{\mathbb{C}} \times{ }^{H^{\mathbb{C}}} V$. Moreover, $\Delta\left(T_{S^{\tau}}^{*} S\right)$ is equal to $\Delta\left(T^{*} S\right) \cap \mathfrak{a}^{*}=\Delta\left(U^{\mathbb{C}} \times{ }^{H^{\mathbb{C}}} V\right) \cap \mathfrak{a}^{*}$ and is therefore likewise a rational convex polyhedral cone.

Proof. See [28. Theorem 7.6] for the first statement. To prove the second statement, observe that the moment map is homogeneous in the cotangent directions and 
therefore $\Delta\left(T_{S^{\tau}}^{*} S\right)$ is equal to the cone spanned by $\Delta(O)$, where $O$ is any open subset of $T_{S^{\tau}}^{*} S$ containing the zero section. Now apply Theorem 8.2 .

Coadjoint orbits and subgroups. Let $M$ be a coadjoint orbit of $U$. In [3] a description was given of the moment polytope of $M$ considered as a Hamiltonian manifold for the action of a subgroup of $U$. In this section we combine the results of [3] with Theorem 3.1 to describe the moment polytope of the real flag variety $M^{\tau}$ relative to the action of a subgroup.

More specifically, let $\tilde{U}$ be a closed connected subgroup of $U$ and denote the inclusion map $\tilde{U} \rightarrow U$ by $f$. Assume that $\tilde{U}$ is $\sigma$-stable and denote the restriction of $\sigma$ to $\tilde{U}$ by $\tilde{\sigma}$. Then we have a decomposition $\tilde{\mathfrak{u}}=\tilde{\mathfrak{k}} \oplus \tilde{\mathfrak{q}}$ analogous to (B.1). Let $\tilde{Q}=\left\{\tilde{u} \tilde{\sigma}(\tilde{u})^{-1} \mid \tilde{u} \in \tilde{U}\right\}$ be the symmetric space and let $\tilde{A}$ be a maximal torus in $\tilde{Q}$ containing 1 . Let $\tilde{T}$ be a maximal torus in $\tilde{U}$ containing $A$ and let $T$ be a maximal torus in $U$ containing $\tilde{T}$. It is not necessarily possible to choose $T$ in such a way that $A=T \cap Q$ is a maximal torus in $Q$, but for simplicity we shall assume this to be the case. (It is certainly possible in the examples we discuss below.) Let $f^{*}: \mathfrak{t}^{*} \rightarrow \tilde{\mathfrak{t}}^{*}$ be the projection induced by $f$. Then $f^{*}$ maps $\mathfrak{a}^{*}$ onto $\tilde{\mathfrak{a}}^{*}$.

We assume the orbit $M$ to be symmetric, i.e. $M=K \lambda$ with $\lambda \in \mathfrak{a}^{*}$, so that $M^{\tau}=K \lambda$, and we consider $M$ as a Hamiltonian $(\tilde{U}, \tilde{\sigma})$-manifold. The following inequalities for $\Delta\left(M^{\tau}\right)$ are now an immediate consequence of Theorem 3.1] and [3. Theorem 3.2.1].

9.2. Theorem. Let $(\tilde{\lambda}, \lambda) \in \tilde{\mathfrak{a}}_{+}^{*} \times \mathfrak{a}_{+}^{*}$. Then $\tilde{\lambda} \in \Delta\left(M^{\tau}\right)$ if and only if

$$
\tilde{w}^{-1} \tilde{\lambda} \in f^{*}\left(w^{-1} \lambda-v \mathcal{C}\right)
$$

for all triples $(\tilde{w}, w, v) \in \tilde{W} \times W \times W_{\text {rel }}$ such that $\tilde{\sigma}_{\tilde{w}}$ is contained in $\phi^{*}\left(v \sigma_{w v}\right)$.

Here $W_{\text {rel }}$ is the relative Weyl set, which is defined as follows. The compatible Weyl set $W_{\text {com }}$ is the set of all $w \in W \operatorname{such}$ that $\operatorname{dim}\left(w \mathfrak{t}_{+} \cap \tilde{\mathfrak{t}}_{+}\right)=\operatorname{dim} \tilde{\mathfrak{t}}_{+}$, where $\mathfrak{t}_{+}$ and $\tilde{\mathfrak{t}}_{+}$are the respective Weyl chambers in $\mathfrak{t}$ and $\tilde{\mathfrak{t}}$. Observe that $W_{\text {com }}$ is stable under left multiplication by $\mathcal{Z}_{W}(\tilde{\mathfrak{t}})$, the centralizer of $\tilde{\mathfrak{t}}$ in $W$. The relative Weyl set is then the set of shortest representatives of the left $\mathcal{Z}_{W}(\tilde{\mathfrak{t}})$-cosets in $W_{\text {com }}$. For each $\tilde{w}, w$ and $v$ the condition (9.1) represents a finite number of linear inequalities. Let $X=U / T$ and $\tilde{X}=\tilde{U} / \tilde{T}$ be the flag varieties of $U$ and $\tilde{U}$, respectively. Then $\sigma_{w} \in H^{2 l(w)}(X)$ denotes the Schubert class associated with $w \in W$ and $\phi: \tilde{X} \hookrightarrow X$ the embedding induced by $f$. We say that a Schubert class $\tilde{\sigma}_{\tilde{w}}$ is contained in a cohomology class $c$ if it appears in the expansion of $c$ in terms of the Schubert basis. See [3] for a more detailed discussion.

Let $\Delta\left(T_{K}^{*} U\right) \subseteq \tilde{\mathfrak{a}}_{+}^{*} \times \mathfrak{a}_{+}^{*}$ be the moment cone of the conormal bundle $T_{K}^{*} U$, considered as a $\tilde{U} \times U$-manifold. The polytopes $\Delta\left(M^{\tau}\right)$ can be obtained by slicing this cone horizontally. The inequalities (9.1) also describe $\Delta\left(T_{K}^{*} U\right)$ and thus Theorem 9.2 can be regarded as a more explicit version of Theorem 9.1 for $S=U$.

The maximal torus. The easiest special case of Theorem 9.2 is that of $\tilde{U}=T$, the maximal torus of $U$. Here of course $\tilde{T}=T, \tilde{W}=\{1\}$ and $f^{*}$ is the identity map. Furthermore, the flag variety $\tilde{X}$ is a point, so $\phi^{*}$ is the trivial homomorphism $H \bullet(X, \mathbb{Z}) \rightarrow \mathbb{Z}$. It follows that $\phi^{*}\left(v \sigma_{w v}\right) \neq 0$ if and only if $v=w^{-1}$. Hence for every $\lambda \in \mathfrak{a}_{+}^{*}$ the points $\tilde{\lambda}$ in the moment polytope of the real flag variety $M^{\tau}$ are described by the inequalities $w \tilde{\lambda} \in \lambda-\mathcal{C}$, where $w$ ranges over $W$. By Lemma B.3 
this is equivalent to $w \tilde{\lambda} \in \lambda-\mathcal{C}^{\mathfrak{a}}$ for all $w \in W$. This implies Kostant's result $\Delta\left(M^{\tau}\right)=$ hull $W^{\mathfrak{a}} \lambda$, which was discussed in Example 3.4 .

Products of orbits. Let us apply Theorem 9.2 to the diagonal inclusion of a group into two copies of itself. We denote the "small" group by $U$ and the "large" group by $U^{2}$. We take the involution on $U^{2}$ to be the direct product of $\sigma$ with itself; the diagonal $f(U)$ is obviously stable under this involution. The projection $f^{*}:\left(\mathfrak{t}^{*}\right)^{2} \rightarrow \mathfrak{t}^{*}$ is the addition map and the homomorphism $\phi^{*}: H^{\bullet}(X, \mathbb{Z})^{\otimes 2} \cong$ $H^{\bullet}\left(X^{2}, \mathbb{Z}\right) \rightarrow H^{\bullet}(X, \mathbb{Z})$ is the cup product. Furthermore, the small Weyl chamber $\mathfrak{t}_{+}$is contained diagonally in the big chamber $\mathfrak{t}_{+}^{2}$ and hence $W_{\text {rel }}=\{1\}$. We obtain the following result from Theorem 9.2 .

9.3. Theorem. Let $\lambda, \mu$ and $\nu \in \mathfrak{a}_{+}^{*}$. Then $\nu \in \Delta(K \lambda \times K \mu)$ if and only if

$$
w^{-1} \nu \leq u^{-1} \lambda+v^{-1} \mu
$$

for all $u, v$ and $w \in W$ such that $\sigma_{w}$ is contained in $\sigma_{u} \cup \sigma_{v}$.

Here $\leq$ denotes the partial ordering on $\mathfrak{a}^{*}$ defined by the cone $\mathcal{C}^{\mathfrak{a}}$. There is also a more "economical" version of this result, in which the vector inequalities (9.2) are replaced by scalar inequalities, and the conditions on Schubert classes for the general flag variety are replaced with conditions on Schubert classes for the Grassmannians of $U$; cf. [3, Theorem 4.2.1]. See Example 5.14 for a discussion of the $G$-orbits on $K \lambda \times K \mu$. The Ky Fan inequalities of Tam [29] are a special case of $(9.2)$.

For $U=\mathbf{S U}(n), \sigma=$ complex conjugation and $K=\mathbf{S O}(n)$ Theorem 9.3 was also observed by Fulton as a corollary of a result of Klyachko [18]. In this case we have $\mathfrak{a}=\mathfrak{t}$ and so the moment polytope of $M$ is the same as that of $M^{\tau}$. Theorem 9.3 then represents a complete list of inequalities satisfied by the eigenvalues of a sum $A+B$ of symmetric matrices in terms of the eigenvalues of $A$ and $B$. Such inequalities were first derived by Weyl. See Fulton's paper [7] for a survey.

Isotropic flags. We discuss Theorem 9.3 in more detail for $U=\mathbf{S U}(n)$, where we take the involution to be the inner automorphism defined by

$$
I_{p q}=\left(\begin{array}{cc}
-I_{p} & 0 \\
0 & I_{q}
\end{array}\right)
$$

with $p+q=n$ and $p \leq q$. We start by giving an explicit description of the varieties $K \lambda$. Observe that $U^{\sigma}=K=\mathbf{S}(\mathbf{U}(p) \times \mathbf{U}(q)), G=\mathbf{S U}(p, q)$, and that $\mathfrak{q}$ consists of all matrices of the form

$$
\left(\begin{array}{cc}
0 & Z \\
-Z^{*} & 0
\end{array}\right)
$$

where $Z$ is a complex $p \times q$-matrix. The action of $K$ is given by

$$
(C, D) Z=C Z D^{*}
$$

for $C \in \mathbf{U}(p)$ and $D \in \mathbf{U}(q)$. The restricted root system is $\mathbf{B} \mathbf{C}_{p}$ if $p<q$ and $\mathbf{C}_{p}$ if $p=q$. (See e.g. [22, Ch. VII, §2].) Consider the antidiagonal $p \times p$-matrices

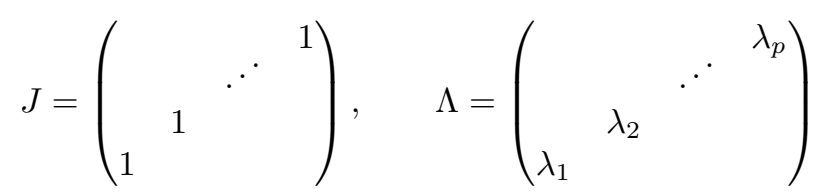


with $\lambda_{1}, \lambda_{2}, \ldots, \lambda_{p}$ real. Then a maximal abelian subspace $\mathfrak{a}$ of $\mathfrak{q}$ is given by all $n \times n$-matrices of the form

$$
\lambda=\left(\begin{array}{ccc}
p & q-p & p \\
0 & 0 & -J \Lambda J \\
0 & 0 & 0 \\
\Lambda & 0 & 0
\end{array}\right){ }_{p}^{p}
$$

and $\mathfrak{a}_{+}^{*} \cong \mathfrak{a}_{+}$is given by $\lambda_{1} \geq \lambda_{2} \geq \cdots \geq \lambda_{p} \geq 0$. The unitary matrix

$$
E=\frac{1}{\sqrt{2}}\left(\begin{array}{ccc}
I & 0 & i J \\
0 & \sqrt{2} I & 0 \\
J & 0 & -i I
\end{array}\right) \in \mathbf{U}(n)
$$

diagonalizes $\mathfrak{a}$ and maps $\mathfrak{a}_{+}$into the standard chamber $\mathfrak{t}_{+}$,

$$
\operatorname{Ad}(E) \lambda=\left(\begin{array}{ccc}
i J \Lambda & 0 & 0 \\
0 & 0 & 0 \\
0 & 0 & -i \Lambda J
\end{array}\right) .
$$

Now fix $\lambda \in \mathfrak{a}_{+}^{*}$ and let $d=\left(d_{1}, d_{2}, \ldots, d_{k}\right)$ be the partition of $p$ determined by the conditions

$$
\lambda_{1}=\cdots=\lambda_{d_{1}}>\lambda_{d_{1}+1}=\cdots=\lambda_{d_{1}+d_{2}}>\cdots>\lambda_{d_{1}+\cdots+d_{k-1}}=\cdots=\lambda_{p} .
$$

Let $\mathcal{F}_{d}$ be the manifold of complex flags

$$
F: V_{0} \subseteq V_{1} \subseteq \cdots \subseteq V_{2 k+1} \subseteq V_{2 k+2}
$$

in $\mathbb{C}^{n}$ such that $V_{0}=\{0\}, V_{2 k+2}=\mathbb{C}^{n}$ and

$$
\operatorname{dim} V_{i}-\operatorname{dim} V_{i-1}= \begin{cases}d_{i} & \text { if } 1 \leq i \leq k \\ q-p & \text { if } i=k+1 \\ d_{2 k+2-i} & \text { if } k+2 \leq i \leq 2 k+1\end{cases}
$$

Then $\operatorname{Ad}(E) \lambda$ has the same stabilizer as the "standard" flag

$$
F_{0}:\{0\} \subseteq \mathbb{C}^{d_{1}} \subseteq \mathbb{C}^{d_{1}+d_{2}} \subseteq \cdots \subseteq \mathbb{C}^{p} \subseteq \mathbb{C}^{q} \subseteq \mathbb{C}^{q+d_{k}} \subseteq \cdots \subseteq \mathbb{C}^{p+q-d_{1}} \subseteq \mathbb{C}^{n} .
$$

It follows that there is a unique $U^{\mathbb{C}}$-equivariant isomorphism $\psi: \mathcal{F}_{d} \rightarrow U \lambda$ which sends $E^{-1} F_{0}$ to $\lambda$. The flag perpendicular to $F \in \mathcal{F}_{d}$ is defined by

$$
F^{\perp}:\{0\} \subseteq V_{2 k+1}^{\perp} \subseteq V_{2 k}^{\perp} \subseteq \cdots \subseteq V_{1}^{\perp} \subseteq \mathbb{C}^{n},
$$

where the orthogonal complements are taken relative to the nondegenerate Hermitian form $\langle v, w\rangle_{p q}=\left\langle E I_{p q} E^{-1} v, w\right\rangle$, which has signature $q-p$. Then $F^{\perp} \in \mathcal{F}_{d}$, so $\perp$ defines an involution on $\mathcal{F}_{d}$ and we obtain the diagram

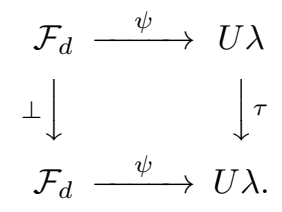

9.4. Lemma. The diagram (9.6) commutes. Hence $K \lambda$ is $G$-equivariantly diffeomorphic to the manifold of flags $\{0\} \subseteq V_{1} \subseteq \cdots \subseteq V_{k}$ in $\mathbb{C}^{n}$ such that $V_{i}$ is $\langle\cdot, \cdot\rangle_{p q}$-isotropic and $\operatorname{dim} V_{i}=\sum_{j=1}^{i} d_{j}$ for $i \leq k$. 
Proof. The first statement follows easily from the fact that $F_{0}=F_{0}^{\perp}$ and

$$
\left\langle E I_{p q} E^{-1} A v, A E I_{p q} E^{-1} w\right\rangle_{p q}=\langle v, w\rangle_{p q}
$$

for all $A \in \mathbf{U}(n)$. To prove the second statement, recall that $K \lambda=(U \lambda)^{\tau}$, which implies that $K \lambda$ is isomorphic to the variety of self-perpendicular flags in $\mathcal{F}_{d}$. Now observe that if $F=F^{\perp}$, then the truncated flag $F_{\leq k}:\{0\} \subseteq V_{1} \subseteq \cdots \subseteq V_{k}$ is isotropic and $F$ is completely determined by $F_{\leq k}$.

Every matrix of the form (9.3) is in the $K$-orbit of a unique matrix $\lambda$ in $\mathfrak{a}_{+}^{*}$. The numbers $\lambda_{1} \geq \lambda_{2} \geq \cdots \geq \lambda_{p} \geq 0$ in (9.5) are known as the singular values. Let us call the vector $\left(\lambda_{1}, \lambda_{2}, \ldots, \lambda_{p}\right)$ corresponding to a $p \times q$-matrix $Z$ the singular spectrum of $Z$. For $\lambda$ and $\mu \in \mathfrak{a}_{+}^{*}$ the polytope $\Delta(K \lambda \times K \mu)$ is the collection of singular spectra of all complex matrices of the form $A+B$, where $A$ and $B$ are $p \times q$-matrices with singular spectra $\lambda$ and $\mu$, respectively. (One gets a corresponding result for singular values of real $p \times q$-matrices by considering $U=\mathbf{S O}(p+q)$ with the inner involution given by $I_{p q}$. Although the abelian subspace $\mathfrak{a}$ is the same here as in the complex case, the root systems are different and one finds different polytopes.) We wish to thank W. Fulton for pointing out the relationship with singular values. See also his preprint [8], which appeared after the first version of this paper.

In the Lorentzian case $p=1$ it is easy to find the inequalities for $\Delta(K \lambda \times K \mu)$ directly. It is plain from (9.4) or Lemma 9.4 that the orbits of $K$ on $\mathfrak{q}$ are $2 n-3$ spheres. The restricted Weyl chamber is a half-line, and the quotient map $\mathfrak{q}^{*} \rightarrow \mathbb{R}_{\geq 0}$ assigns to a sphere its radius. The moment "polytope" of a product of two spheres $K \lambda$ and $K \mu$ is therefore simply the interval between $|\lambda-\mu|$ and $\lambda+\mu$. According to Example 5.14, there are two $G$-orbits on $S^{2 n-3} \times S^{2 n-3}$ corresponding to the two elements of $W^{\mathfrak{a}}$, namely the diagonal and its complement. The subpolytopes associated with these orbits are $\{\lambda+\mu\}$, resp. the whole interval.

Let us also write out the case $p=q=2$. The inequalities (9.2) can be enumerated explicitly as explained in [3] or [7]. They are of the form

$$
\sum_{k \in \mathcal{K}} \nu_{k} \leq \sum_{i \in \mathcal{I}} \lambda_{i}+\sum_{j \in \mathcal{J}} \mu_{j}
$$

where $\mathcal{I} \mathcal{J K}$ range over all triples of ordered subsets of $\{1,2,3,4\}$ listed in Table 1. For instance, the triple $(1,2)(2,4)(2,4)$ encodes the inequality $\nu_{2}+\nu_{4} \leq \lambda_{1}+$ $\lambda_{2}+\mu_{2}+\mu_{4}$. For each triple $\mathcal{I} \mathcal{J K}$ there is a similar inequality corresponding to $\mathcal{J} \mathcal{I K}$. To save space, for each $\mathcal{I}, \mathcal{J}, \mathcal{K}$ only one of the triples $\mathcal{I} \mathcal{J K}$ and $\mathcal{J} \mathcal{I} \mathcal{K}$ is listed in the table. Next to each triple $\mathcal{I} \mathcal{J K}$ is listed the dual triple $\mathcal{I}^{*} \mathcal{J}^{*} \mathcal{K}^{*}$. (If $\mathcal{I}=\left(i_{1}<i_{2}<\cdots<i_{k}\right)$ is a $k$-tuple of integers between 1 and $n$, and $\{1,2, \ldots, n\} \backslash$ $\mathcal{I}=\left(i_{k+1}<i_{k+2}<\cdots<i_{n}\right)$ is the complementary $n-k$-tuple, then $\mathcal{I}^{*}$ is defined as $\left(n+1-i_{n}, n+1-i_{n-1}, \ldots, n+1-i_{k+1}\right)$. The inequality associated with $\mathcal{I}^{*} \mathcal{J}^{*} \mathcal{K}^{*}$ is obtained from the one associated with $\mathcal{I} \mathcal{J K}$ by substituting $\lambda \rightarrow \lambda^{*}, \mu \rightarrow \mu^{*}$, $\nu \rightarrow \nu^{*}$, where $\lambda^{*}=-w_{0} \lambda$.) For those triples which are self-dual we have omitted the corresponding entry in the second column.

We get the inequalities for $\Delta\left(M^{\tau}\right)$ by substituting $\lambda_{1}+\lambda_{4}=0$ and $\lambda_{2}+\lambda_{3}=0$ (plus similar equalities for $\mu$ and $\nu$ ) into the inequalities for $\Delta(M)$. Most inequalities turn out to be redundant. In particular, every inequality becomes identical to its dual inequality, and all non-self-dual inequalities with $\# \mathcal{I}=\# \mathcal{J}=\# \mathcal{K}=2$ drop out. Here is a complete list, consisting of eighteen inequalities (besides the obvious 
TABLE 1. Horn-Klyachko inequalities for SU(4)

\begin{tabular}{|c|c|}
\hline triple & dual triple \\
\hline \hline$(1)(1)(1)$ & $(1,2,3)(1,2,3)(1,2,3)$ \\
$(1)(2)(2)$ & $(1,2,3)(1,2,4)(1,2,4)$ \\
$(1)(3)(3)$ & $(1,2,3)(1,3,4)(1,3,4)$ \\
$(1)(4)(4)$ & $(1,2,3)(2,3,4)(2,3,4)$ \\
$(2)(2)(3)$ & $(1,2,4)(1,2,4)(1,3,4)$ \\
$(2)(3)(4)$ & $(1,2,4)(1,3,4)(2,3,4)$ \\
\hline
\end{tabular}

\begin{tabular}{|c|c|}
\hline triple & dual triple \\
\hline \hline$(1,2)(1,2)(1,2)$ & \\
$(1,2)(1,3)(1,3)$ & \\
$(1,2)(1,4)(1,4)$ & $(1,2)(2,3)(2,3)$ \\
$(1,2)(2,4)(2,4)$ & \\
$(1,2)(3,4)(3,4)$ & \\
$(1,3)(1,3)(1,4)$ & $(1,3)(1,3)(2,3)$ \\
$(1,3)(1,4)(2,4)$ & $(1,3)(2,3)(2,4)$ \\
$(1,3)(2,4)(3,4)$ & \\
$(1,4)(1,4)(3,4)$ & $(2,3)(2,3)(3,4)$ \\
\hline
\end{tabular}

inequalities $\left.\lambda_{1} \geq \lambda_{2} \geq 0, \mu_{1} \geq \mu_{2} \geq 0, \nu_{1} \geq \nu_{2} \geq 0\right)$ :

$$
\begin{aligned}
& \nu_{1} \leq \lambda_{1}+\mu_{1} \quad \nu_{1}+\nu_{2} \leq \lambda_{1}+\lambda_{2}+\mu_{1}+\mu_{2} \\
& \nu_{2} \leq\left\{\begin{array}{l}
\lambda_{1}+\mu_{2} \\
\lambda_{2}+\mu_{1}
\end{array} \quad \nu_{1}-\nu_{2} \leq\left\{\begin{array}{l}
\lambda_{1}+\lambda_{2}+\mu_{1}-\mu_{2} \\
\lambda_{1}-\lambda_{2}+\mu_{1}+\mu_{2}
\end{array}\right.\right. \\
& \nu_{2} \geq\left\{\begin{array}{l}
-\lambda_{1}+\mu_{2} \\
\lambda_{2}-\mu_{1}
\end{array} \quad \nu_{1}-\nu_{2} \geq\left\{\begin{array}{l}
-\lambda_{1}-\lambda_{2}+\mu_{1}-\mu_{2} \\
\lambda_{1}-\lambda_{2}-\mu_{1}-\mu_{2}
\end{array}\right.\right. \\
& \nu_{1} \geq\left|\lambda_{1}-\mu_{1}\right| \quad \nu_{1}+\nu_{2} \geq\left|\lambda_{1}+\lambda_{2}-\mu_{1}-\mu_{2}\right| \\
& \nu_{1} \geq\left|\lambda_{2}-\mu_{2}\right| \quad \nu_{1}+\nu_{2} \geq\left|\lambda_{1}-\lambda_{2}-\mu_{1}+\mu_{2}\right| .
\end{aligned}
$$

See Figure 1 for an example. Also shown, in dark, is the subpolytope $\Delta\left(\overline{\mathcal{O}}_{w}\right)$, where $w$ is the product of the simple reflections $s_{1}$ and $s_{2}$ in $W^{\mathfrak{a}}$. The simple roots are denoted by $\alpha_{1}$ and $\alpha_{2}$ and the corresponding fundamental weights by $\pi_{1}$ and $\pi_{2}$.

An unsolved problem. As is obvious from the above examples, the inequalities of Theorem 9.2 are in general highly overdetermined. We believe it should be possible to find a complete set of inequalities which uses only data involving the restricted Weyl groups and cohomology classes on real flag varieties.

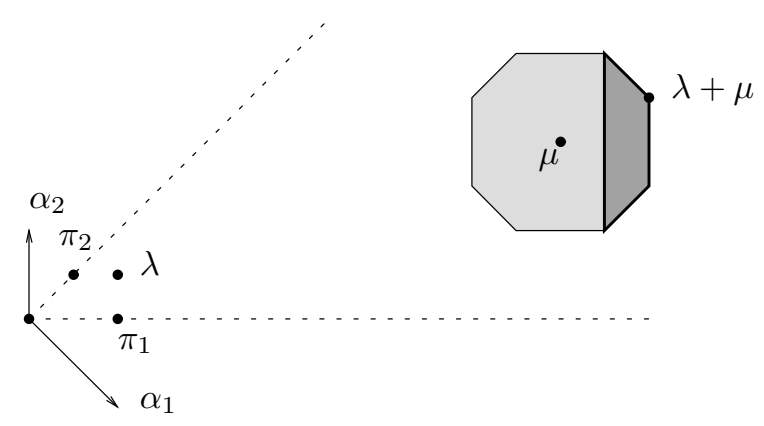

FiguRE 1. Moment polytopes of $K \lambda \times K \mu$ and $\overline{\mathcal{O}}_{w}$ with $K=$ $\mathbf{S}(\mathbf{U}(2) \times \mathbf{U}(2)), \lambda=\frac{1}{2} \pi_{1}+\pi_{2}, \mu=4\left(\pi_{1}+\pi_{2}\right), w=s_{2} s_{1}$ 
9.5. Problem. Does the statement of Theorem 9.2 remain true if we replace $\tilde{W}$, $W$, and $W_{\text {rel }}$ by $\tilde{W}^{\tilde{\mathfrak{a}}}, W^{\mathfrak{a}}$, and $W_{\text {rel }}^{\mathfrak{a}}$, respectively, and complex Schubert classes by $\mathbb{Z} / 2$-Schubert classes on appropriate real flag varieties?

Here $\tilde{W}^{\tilde{\mathfrak{a}}}$ and $W^{\mathfrak{a}}$ are the restricted Weyl groups. The restricted relative Weyl set $W_{\text {rel }}^{\mathfrak{a}}$ is defined in the same way as $W_{\text {rel }}$, except that in the definition $W$ is replaced by $W^{\mathfrak{a}}, \tilde{\mathfrak{t}}$ by $\tilde{\mathfrak{a}}$, and $\mathfrak{t}$ by $\mathfrak{a}$.

In the example of the maximal torus the answer to Problem 9.5 is affirmative. In the $K \lambda \times K \mu$-example it is not very hard to see that the same is true in the low-dimensional cases which we wrote out, although in general the question appears to be nontrivial.

\section{ApPEndix A. ANTI-SYMPleCtiC MAPS}

The results in this appendix can be found in a somewhat weaker form in the papers of Meyer [26] and Duistermaat [6]. Let $(V, \omega)$ be a symplectic vector space. Recall that a complex structure $J$ on $V$ is compatible with $\omega$ if $J \in \mathbf{S p}(V, \omega)$ and the symmetric bilinear form $\omega(\cdot, J \cdot)$ is positive definite. Let $\mathcal{J}(\omega)$ be the set of complex structures on $V$ that are compatible with $\omega$ and $\operatorname{Inn}(V)$ the set of (positive definite) inner products on $V$. Recall that we have maps $\iota: \mathcal{J}(\omega) \rightarrow \operatorname{Inn}(V)$ and $\pi: \operatorname{Inn}(V) \rightarrow \mathcal{J}(\omega)$ defined by

$$
\iota(J)(v, w)=\omega(v, J w), \quad \pi(\beta)=\left(-A_{\beta}^{2}\right)^{-1 / 2} A_{\beta},
$$

where for $\beta \in \operatorname{Inn}(V)$ the antisymmetric linear map $A_{\beta}$ is defined by $\omega(v, w)=$ $\beta\left(A_{\beta} v, w\right)$. Furthermore, the map $\iota$ is injective and $\pi$ is a left-inverse of $\iota$. It follows that $\pi$ is a deformation retraction and that $\mathcal{J}(\omega)$ is contractible. (See McDuff and Salamon [24, §2.5].)

Now let $\mathcal{G}$ be a Lie group with a real character $\varepsilon: \mathcal{G} \rightarrow \mathbb{R}^{\times}$and suppose that $\mathcal{G}$ acts linearly and sesquisymplectically on $V$ in the sense that $g^{*} \omega=\varepsilon(g) \omega$ for $g \in \mathcal{G}$. Define an action of $\mathcal{G}$ on $\mathcal{J}(\omega)$ by

$$
g \cdot J=\varepsilon(g) g \circ J \circ g^{-1} .
$$

Then $J$ is invariant for this action if $J \circ g=\varepsilon(g) g \circ J$. Let $\mathcal{G}$ act on $\operatorname{Inn}(V)$ by $(g \cdot \beta)(v, w)=\beta(g v, g w)$. The proof of the following result is straightforward.

A.1. Lemma. The maps $\iota$ and $\pi$ are $\mathcal{G}$-equivariant. Hence, if $\mathcal{G}$ is compact, $\mathcal{J}(\omega)^{\mathcal{G}}$ is contractible and nonempty.

Of course, if $\mathcal{G}$ is compact, $\varepsilon$ takes values in $\{ \pm 1\}$. Let us denote the kernel of $\varepsilon$ by $\mathcal{G}_{1}$.

A.2. Lemma. Assume that $\mathcal{G}$ is compact. Let $g \in \mathcal{G}$ be antisymplectic. Then the following conditions are equivalent.

(i) $g$ is an involution.

(ii) All eigenvalues of $g$ are real.

(iii) $V^{g}$ is a Lagrangian subspace.

If any of these conditions is satisfied, then $\mathcal{G} \cong \mathcal{G}_{1} \rtimes\{ \pm 1\}$ and $V^{g}$ is totally real with respect to any $\mathcal{G}$-invariant almost complex structure.

Proof. It is obvious that (i) implies (国).

Assume that (国) holds. According to Lemma A.1 there exists $J \in \mathcal{J}(\omega)^{\mathcal{G}}$. Then $g J=-J g$. Moreover, the eigenvalues of $g$ are \pm 1 because $g$ is orthogonal relative 
to the inner product $\iota(J)$. Let $\lambda= \pm 1$ be an eigenvalue of $g$ with eigenvector $e \in V$ and put $f=J e$. Then

$$
g f=g J e=-J g e=-\lambda f=\mp f .
$$

Let $W$ be the real span of $e$ and $f$. Then $W$ is a Hermitian subspace of $W$. Since $g$ is antisymplectic and preserves $W$, it preserves the symplectic subspace $W^{\omega}=W^{\perp}$. By induction on the dimension we may assume that the fixed points of $\left.g\right|_{W \perp}$ form a Lagrangian subspace. On the other hand, it is clear that $\left.g\right|_{W}$ fixes either $e$ or $f$, depending on the value of $\lambda$. This implies (iii).

Now suppose that (iii) holds. Let $\left\{e_{1}, e_{2}, \ldots, e_{n}\right\}$ be a basis of $V^{g}$ and put $f_{i}=J e_{i}$. It follows from (A.1) that $f_{i}$ has eigenvalue -1 and therefore

$$
\left\{e_{1}, f_{1}, e_{2}, f_{2}, \ldots, e_{n}, f_{n}\right\}
$$

is a (symplectic) basis of $V$. Hence (ii) holds.

If $g \in \mathcal{G} \backslash \mathcal{G}_{1}$ is an involution, the exact sequence $\mathcal{G}_{1} \hookrightarrow \mathcal{G} \rightarrow\{ \pm 1\}$ splits and hence $\mathcal{G} \cong \mathcal{G}_{1} \rtimes\{ \pm 1\}$. The fact that $V^{g}$ is totally real follows from the following trivial observation: let $W$ be a (real) vector space with a complex structure $J$ and let $\tau$ be an involution which is antiholomorphic in the sense that $\tau J=-J \tau$. Then the subspaces $W^{\tau}$ and $W^{-\tau}=J W^{\tau}$ are totally real, $\tau$ is complex conjugation with respect to $W^{\tau}$, and $W=W^{\tau} \oplus J W^{\tau} \cong\left(W^{\tau}\right)^{\mathbb{C}}$.

Now let $(M, \omega)$ be a connected symplectic manifold and assume that $\mathcal{G}$ acts sesquisymplectically on $M$, i.e. $g^{*} \omega=\varepsilon(g) \omega$ for $g \in \mathcal{G}$. Let us denote by $\mathcal{J}(M, \omega)$ the space of almost complex structures on $M$ which are compatible with $\omega$. As before, $\mathcal{G}$ acts in a natural way on $\mathcal{J}(M, \omega)$.

A.3. Lemma. Assume that $\mathcal{G}$ acts properly on $M$.

(i) $\mathcal{J}(M, \omega)^{\mathcal{G}}$ is nonempty and connected.

(ii) If there exist $g \in \mathcal{G} \backslash \mathcal{G}_{1}$ and $m \in M$ such that $g$ fixes $m$ and has only real eigenvalues at $m$, then $g$ is an involution and $M^{g}$ is a Lagrangian submanifold. Moreover, $M^{g}$ is totally real with respect to any $\mathcal{G}$-invariant almost complex structure.

Proof. (这) follows from Lemma A.1 by a standard argument as in [24, §2.6]. (目) is proved by applying Lemma A.2 to the stabilizer subgroup $\mathcal{G}_{m}$ (which is compact and contains $g$ ) acting on the tangent space $T_{m} M$.

\section{Appendix B. Symmetric Pairs}

This appendix is a collection of elementary facts concerning the symmetric pair $(U, K)$. In large part, this is well-known material culled from Helgason [12] and Loose 22. Let $U$ be a compact connected Lie group with involution $\sigma$ and let $K=\left(U^{\sigma}\right)_{0}$. Let

$$
Q=\left\{u \sigma(u)^{-1} \mid u \in U\right\}
$$

be the submanifold of symmetric elements. Put $\mathfrak{q}=T_{1} Q$. Then $Q=\exp \mathfrak{q}$ and the map $U \rightarrow Q$ which sends $u$ to $u \sigma(u)^{-1}$ induces an isometry from the symmetric space $U / U^{\sigma}$ onto $Q$. (See e.g. Chapters II and VI in [22].) The Lie algebra of $U$ decomposes into the \pm 1 -eigenspaces for the involution $\sigma$,

$$
\mathfrak{u}=\mathfrak{k} \oplus \mathfrak{q}
$$

and dually we have $\mathfrak{u}^{*}=\mathfrak{k}^{*} \oplus \mathfrak{q}^{*}$. Let $A$ be a maximal torus (connected flat submanifold) in $Q$ containing 1 and let $\mathfrak{a}=T_{1} A$. Then $\mathfrak{a}$ is a maximal abelian 
subspace of $\mathfrak{q}$ and $A=\exp \mathfrak{a}$. Clearly $A$ is stable under $\sigma$. The fixed-point group ("real part") is

$$
A^{\sigma}=A[2]=\left\{a \mid a^{2}=1\right\},
$$

the subgroup of 2 -torsion elements. Hence $\# A^{\sigma}=2^{r}$, where $r=\operatorname{dim} A$, the rank of the symmetric space $Q$. The following assertion is a straightforward corollary of the $K A K$-decomposition, Theorem 8.6 in [12, Ch. VII].

B.1. Lemma. $U^{\sigma}=K A[2]=A[2] K$.

The factors $K$ and $A[2]$ usually have a nontrivial intersection, so this product is rarely semidirect. Now choose a maximal torus $T$ of $U$ such that $T \supseteq A$. Such a torus is always $\sigma$-stable. Let $K^{\prime}=\mathcal{Z}_{K}(A)$, the centralizer of $A$ in $K$. Then $T^{\prime}=\left(K^{\prime}\right)_{0} \cap T$ is a maximal torus of (the identity component of) $K^{\prime}$. Moreover,

$$
T=T^{\prime} A
$$

and $T^{\prime} \cap A \subseteq A[2]$. (See Proposition 3.3 in 22, Ch. VI].) From (B.1) and (B.2) we get the direct-sum decomposition

$$
\mathfrak{t}=\mathfrak{t}^{\prime} \oplus \mathfrak{a}
$$

Let $R$ be the root system of $(U, T)$. Since $\sigma$ is an automorphism and $\mathfrak{t}$ is $\sigma$-stable, $\sigma$ acts on $R$. Define

$$
R^{\prime}=\{\alpha \in R \mid \sigma(\alpha)=\alpha\}, \quad R^{\mathfrak{a}}=\left\{\left.\alpha\right|_{\mathfrak{a}} \mid \alpha \notin R^{\prime}\right\} .
$$

Then $R^{\prime}$ is the set of roots which vanish on $\mathfrak{a}$. It is a root subsystem of $R$, namely the root system of $\left(K^{\prime}, T^{\prime}\right)$, and $R^{\mathfrak{a}}$ is a (not necessarily reduced) root system in $\mathfrak{a}^{*}$, known as the system of restricted roots of the symmetric pair $(\mathfrak{u}, \mathfrak{k})$. We define sets of positive roots in $R$ and $R^{\mathfrak{a}}$ as follows: fix sets of positive roots $R_{+}^{\prime}$ in $R^{\prime}$ and $R_{+}^{\mathfrak{a}}$ in $R^{\mathfrak{a}}$. Then

$$
R_{+}=R_{+}^{\prime} \cup\left\{\alpha \in R|\alpha|_{\mathfrak{a}} \in R_{+}^{\mathfrak{a}}\right\}
$$

is a system of positive roots in $R$. Let

$$
\mathfrak{t}_{+}=\left\{\xi \in \mathfrak{t} \mid \alpha(\xi) \geq 0 \text { for } \alpha \in R_{+}\right\} \quad \text { and } \quad \mathfrak{a}_{+}=\left\{\xi \in \mathfrak{t} \mid \alpha(\xi) \geq 0 \text { for } \alpha \in R_{+}^{\mathfrak{a}}\right\}
$$

be the associated Weyl chambers in $\mathfrak{t}$, resp. $\mathfrak{a}$. Then $\mathfrak{t}_{+}$is a fundamental domain for the $U$-action on $\mathfrak{u}$ and $\mathfrak{a}_{+}$is a fundamental domain for the $K$-action on $\mathfrak{q}$. Let $\mathfrak{t}_{+}^{*} \subseteq \mathfrak{t}^{*}$ and $\mathfrak{a}_{+}^{*} \subseteq \mathfrak{a}^{*}$ be the corresponding dual Weyl chambers. Because of the way the positive roots in $\mathfrak{t}$ and $\mathfrak{a}$ are related we have the following equalities.

B.2. Lemma. $\mathfrak{a}_{+}=\mathfrak{t}_{+} \cap \mathfrak{q}$ and $\mathfrak{a}_{+}^{*}=\mathfrak{t}_{+}^{*} \cap \mathfrak{q}^{*}$.

Likewise, let $\mathcal{C}$ be the root cone, i.e. the cone in $\mathfrak{t}^{*}$ spanned by the positive roots $R_{+}$, and let $\mathcal{C}^{\mathfrak{a}}$ be the restricted root cone, i.e. the cone in $\mathfrak{a}^{*}$ spanned by $R_{+}^{\mathfrak{a}}$. Then $\mathcal{C}$ is the dual cone of $\mathfrak{t}_{+}$and $\mathcal{C}^{\mathfrak{a}}$ is the dual cone of $\mathfrak{a}_{+}$. The root cones are related as follows.

B.3. Lemma. $\mathcal{C}^{\mathfrak{a}}=\mathcal{C} \cap \mathfrak{a}^{*}$.

Proof. The inclusion $\mathcal{C}^{\mathfrak{a}} \subseteq \mathcal{C} \cap \mathfrak{a}^{*}$ is obvious. The reverse inclusion follows easily from the fact that $\alpha(\xi)=\frac{1}{2}(\alpha+\sigma(\alpha))(\xi)$ for $\alpha \in R$ and $\xi \in \mathfrak{a}$.

Finally we recall the relationship between the Weyl groups of the root systems $R, R^{\prime}$ and $R^{\mathfrak{a}}$, which we denote by $W, W^{\prime}$ and $W^{\mathfrak{a}}$, respectively. Observe that $\sigma$ acts in a natural way on $W$ because $\mathfrak{t}$ is $\sigma$-stable. 
B.4. Lemma. (i) $W^{\prime} \cong \mathcal{Z}_{W}(\mathfrak{a}), W^{\sigma}=\mathcal{N}_{W}(\mathfrak{a})$, and $W^{\mathfrak{a}} \cong W^{\sigma} / W^{\prime}$.

(ii) $(U \lambda)^{A} \cap \mathfrak{q}^{*}=U \lambda \cap \mathfrak{a}^{*}=W \lambda \cap \mathfrak{a}^{*}=W^{\mathfrak{a}} \lambda$ for all $\lambda \in \mathfrak{a}^{*}$.

(iii) (hull $W \lambda$ ) $\cap \mathfrak{a}^{*}=$ hull $W^{\mathfrak{a}} \lambda$ for all $\lambda \in \mathfrak{a}^{*}$.

Here " $\mathcal{Z}$ " denotes the centralizer of a subalgebra, "N $\mathcal{N}$ " its normalizer, and "hull" denotes the convex hull of a subset of a real vector space.

Proof. The isomorphisms $W^{\prime} \cong \mathcal{Z}_{W}(\mathfrak{a})$ and $W^{\mathfrak{a}} \cong \mathcal{N}_{W}(\mathfrak{a}) / \mathcal{Z}_{W}(\mathfrak{a})$ are in 12, p. 325]. If $w \in W^{\sigma}$, then $\sigma(w \xi)=\sigma(w) \sigma(\xi)=-w \xi$ for all $\xi \in \mathfrak{a}$, and therefore $w \in \mathcal{N}_{W}(\mathfrak{a})$. Conversely, if $w \in \mathcal{N}_{W}(\mathfrak{a})$, then $w$ preserves the decomposition (B.3). (This follows for instance from the fact that the decompositions (B.1) and (B.3) are orthogonal with respect to the Killing form.) Now let $\xi \in \mathfrak{t}$ and write $\xi=\xi^{+}+\xi^{-}$ with $\xi^{+} \in \mathfrak{t}^{\prime}$ and $\xi^{-} \in \mathfrak{a}$. Then

$\sigma(w) \xi=\sigma(w)\left(\xi^{+}+\xi^{-}\right)=\sigma\left(w \sigma\left(\xi^{+}\right)+w \sigma\left(\xi^{-}\right)\right)=\sigma\left(w \xi^{+}-w \xi^{-}\right)=w \xi^{+}+w \xi^{-}=w \xi$

and hence $\sigma(w)=w$. The conclusion is that $\mathcal{N}_{W}(\mathfrak{a})=W^{\sigma}$. This proves (i).

The first equality in (iii) follows easily from the fact that $A$ is maximal abelian in $Q$. To prove the second equality, observe that $U \lambda \cap \mathfrak{a}^{*} \subseteq U \lambda \cap \mathfrak{t}^{*}=W \lambda$, so $U \lambda \cap \mathfrak{a}^{*} \subseteq W \lambda \cap \mathfrak{a}^{*}$. The last equality is Corollary 8.9 of [12, Ch. VII].

The inclusion hull $W^{\mathfrak{a}} \lambda \subseteq$ (hull $\left.W \lambda\right) \cap \mathfrak{a}$ follows from (iii). The reverse inclusion follows from the fact that

$$
\text { hull } W \lambda=\bigcap_{w \in W} w(\lambda-\mathcal{C}) \quad \text { and } \quad \text { hull } W^{\mathfrak{a}} \lambda=\bigcap_{w \in W^{\mathfrak{a}}} w\left(\lambda-\mathcal{C}^{\mathfrak{a}}\right),
$$

combined with Lemma B.3.

\section{Appendix C. Notation}

$U ; \sigma$

$U^{\sigma} ; K$

$Q ; \mathfrak{q}$

$\mathfrak{u}=\mathfrak{k} \oplus \mathfrak{q}$

$G ; \theta$

$\mathfrak{g}=\mathfrak{k} \oplus \mathfrak{p}$

$A ; \mathfrak{a}$

$A[2] ; K^{\prime}$

$T^{\prime} ; T=T^{\prime} A$

$\mathfrak{t}^{\prime} ; \mathfrak{t}=\mathfrak{t}^{\prime} \oplus \mathfrak{a}$

$R ; R^{\prime} ; R^{\mathfrak{a}}$

$W ; W^{\prime} ; W^{\mathfrak{a}}$

$w_{0} ; w_{0}^{\prime} ; w_{0}^{\mathfrak{a}}$

$\mathfrak{t}_{+}^{*} ; \mathfrak{a}_{+}^{*}$

$\mathcal{C} ; \mathcal{C}^{\mathfrak{a}}$

$\Lambda_{+}^{*} ; \sigma_{+}$

$V_{\lambda}$

$\gamma_{\mathfrak{u}} ; \gamma_{\mathfrak{g}}$

$(M, \omega)$

$\Phi ; \tau ; M^{\tau}$

$\xi_{M} ; \Phi^{\xi}$

$\Delta(M) ; \Delta\left(M^{\tau}\right)$ compact connected Lie group; involution

fixed-point group; its identity component

space of symmetric elements $u \sigma(u)^{-1}$; its tangent space at 1

eigenspace decomposition of $\mathfrak{u}$ under $\sigma$

real reductive group dual to $U$; Cartan involution

Cartan decomposition of $\mathfrak{g}$, where $\mathfrak{p}=i \mathfrak{q}$

maximal torus in $Q$; its tangent space at 1

2 -torsion elements in $A$; centralizer of $A$ in $K$

maximal torus of $K^{\prime}$; maximal torus of $U$

Cartan subalgebra of $\mathfrak{k}^{\prime}$; Cartan subalgebra of $\mathfrak{u}$

roots of $(\mathfrak{u}, \mathfrak{t})$; roots of $\left(\mathfrak{k}^{\prime}, \mathfrak{t}^{\prime}\right)$; restricted roots

Weyl groups of $R ; R^{\prime} ; R^{\mathfrak{a}}$

longest elements of $W ; W^{\prime} ; W^{\mathfrak{a}}$

fundamental Weyl chambers for $R$; resp. $R^{\mathrm{a}}$

cone spanned by $R_{+}$; resp. $R_{+}^{\mathfrak{a}}$

set of dominant weights; involution on $\mathfrak{t}_{+}^{*}$ induced by $\sigma$ irreducible $U$-module with highest weight $\lambda \in \Lambda_{+}^{*}$

complex conjugations on $\mathfrak{u}^{\mathbb{C}}$ relative to $\mathfrak{u}$; resp. $\mathfrak{g}$

symplectic manifold acted on by $U$

moment map; antisymplectic involution; fixed-point set vector field induced by $\xi \in \mathfrak{u}$; component of $\Phi$ along $\xi$ moment polytopes $\Phi(M) \cap \mathfrak{t}_{+}^{*}$; resp. $\Phi\left(M^{\tau}\right) \cap \mathfrak{t}_{+}^{*}$ 


\section{REFERENCES}

1. M. Atiyah, Convexity and commuting Hamiltonians, Bull. London Math. Soc. 14 (1982), no. $1,1-15$.

2. R. Benedetti and J.-J. Risler, Real algebraic and semi-algebraic sets, Hermann, Paris, 1990.

3. A. Berenstein and R. Sjamaar, Coadjoint orbits, moment polytopes, and the Hilbert-Mumford criterion, J. Amer. Math. Soc., to appear, math.SG/9810125.

4. G. Bredon, Introduction to compact transformation groups, Pure and Applied Mathematics, vol. 46, Academic Press, New York, 1972.

5. M. Brion, Sur l'image de l'application moment, Séminaire d'algèbre Paul Dubreil et MariePaule Malliavin (Paris, 1986) (M.-P. Malliavin, ed.), Lecture Notes in Mathematics, vol. 1296, Springer-Verlag, Berlin, 1987, pp. 177-192.

6. J. J. Duistermaat, Convexity and tightness for restrictions of Hamiltonian functions to fixed point sets of an antisymplectic involution, Trans. Amer. Math. Soc. 275 (1983), no. 1, 417429.

7. W. Fulton, Eigenvalues of sums of Hermitian matrices (after A. Klyachko), Séminaire Bourbaki, 50ème année, exposé no. 845 (Paris, 1997-98), Astérisque, vol. 252, Société Mathématique de France, Paris, 1998, pp. 255-269.

8. _ Eigenvalues, invariant factors, highest weights, and Schubert calculus, preprint, University of Michigan, 1999.

9. V. Guillemin and S. Sternberg, Convexity properties of the moment mapping, Invent. Math. 67 (1982), no. 3, 491-513.

10. (1982), no. 3, 515-538.

11. _ A normal form for the moment map, Differential Geometric Methods in Mathematical Physics (Jerusalem, 1982), Reidel, Dordrecht, 1984, pp. 161-175.

12. S. Helgason, Differential geometry, Lie groups, and symmetric spaces, Academic Press, New York, 1978.

13. J. Hilgert, K.-H. Neeb, and W. Plank, Symplectic convexity theorems and coadjoint orbits, Compositio Math. 94 (1994), no. 2, 129-180.

14. K. Jänich, Differenzierbare G-Mannigfaltigkeiten, Lecture Notes in Mathematics, vol. 59, Springer-Verlag, Berlin, 1968.

15. J. Jurkiewicz, Torus embeddings, polyhedra, $k^{*}$-actions and homology, Dissertationes Math. (Rozprawy Mat.) 236 (1985), 64.

16. F. Kirwan, Cohomology of quotients in symplectic and algebraic geometry, Mathematical Notes, vol. 31, Princeton University Press, Princeton, N.J., 1984.

17. Convexity properties of the moment mapping, III, Invent. Math. 77 (1984), no. 3, $547-552$.

18. A. Klyachko, Stable bundles, representation theory and Hermitian operators, Selecta Math. (N.S.) 4 (1998), no. 3, 419-445.

19. B. Kostant, Quantization and unitary representations. I. Prequantization, Lectures in Modern Analysis and Applications, III (Washington, D.C.) (C. T. Taam, ed.), Lecture Notes in Mathematics, vol. 170, Springer-Verlag, Berlin, 1970, pp. 87-208.

20. On convexity, the Weyl group and the Iwasawa decomposition, Ann. Sci. École Norm. Sup. (4) 6 (1973), 413-455.

21. E. Lerman, E. Meinrenken, S. Tolman, and C. Woodward, Nonabelian convexity by symplectic cuts, Topology 37 (1998), no. 2, 245-259.

22. O. Loos, Symmetric spaces, vols. I and II, W. A. Benjamin, Inc., New York-Amsterdam, 1969.

23. C.-M. Marle, Modèle d'action hamiltonienne d'un groupe de Lie sur une variété symplectique, Rend. Sem. Mat. Univ. Politec. Torino 43 (1985), no. 2, 227-251.

24. D. McDuff and D. Salamon, Introduction to symplectic topology, Oxford Mathematical Monographs, Oxford University Press, New York, 1995.

25. E. Meinrenken and R. Sjamaar, Singular reduction and quantization, Topology 38 (1999), no. 4, 699-762.

26. K. Meyer, Hamiltonian systems with a discrete symmetry, J. Differential Equations 41 (1981), no. $2,228-238$.

27. L. Ness, A stratification of the null cone via the moment map, Amer. J. Math. 106 (1984), no. 6, 1281-1329, with an appendix by D. Mumford. 
28. R. Sjamaar, Convexity properties of the moment mapping re-examined, Adv. Math. 138 (1998), no. 1, 46-91.

29. T.-Y. Tam, A unified extension of two results of Ky Fan on the sum of matrices, Proc. Amer. Math. Soc. 126 (1998), no. 9, 2607-2614.

30. J. Tits, Tabellen zu den einfachen Lie Gruppen und ihren Darstellungen, Lecture Notes in Mathematics, vol. 40, Springer-Verlag, Berlin, 1967.

Department of Mathematics, Cornell University, Ithaca, New York 14853-7901

E-mail address: luis@math.cornell.edu

E-mail address: sjamaar@math.cornell.edu 